\title{
Synthesis of amide-spacered dimers of ursolic and oleanolic acid
}

\author{
Sophie Hoenke ${ }^{1}$, Isabell Wiengarn ${ }^{1}$, Immo Serbian ${ }^{1}$, Ahmed Al-Harrasi ${ }^{2}$ and René Csuk ${ }^{1, *}$ \\ ${ }^{1}$ Martin-Luther-University Halle-Wittenberg, Organic Chemistry, Kurt-Mothes-Str. 2, D-06120 Halle (Saale), \\ Germany \\ ${ }^{2}$ University of Nizwa, Chair of Oman's Medicinal Plants and Marine Natural Products, P.O. Box 33, PC 616, \\ Birkat Al-Mauz, Nizwa, Sultanate of Oman
}

\begin{abstract}
Transdermal therapeutic systems can release drug substances slowly and in a controlled manner from a drug depot. To provide a slow-release form of ursolic and oleanolic acid amide-spacered dimers were synthesized from the parent acids and diamines of variable chain lengths. These dimers were assayed in sulforhodamine B (SRB) assays for their cytotoxicity since as a pre-requisite for their use in slow-release forms these substances must not exert any unfavorable side-effects such as cytotoxicity. As a result of long term incubation up to 96 hours, none of these compounds showed any significant cytotoxicity in sulforhodamine B assays.
\end{abstract}

Keywords: ursolic acid; oleanolic acid; triterpenes; dimers.

\section{Introduction}

In recent years, the search for biologically active compounds has increasingly turned to secondary natural substances. A newly awakened interest consists in the isolation, synthesis and biological investigation of dimeric compounds. Such dimers consist of two identical monomeric basic structures, which are connected to each other by a suitable linker. Some dimers seem to hold increased biological activity as compared to the corresponding monomers ${ }^{1-5}$. The formation of dimers, trimers, etc... is a common phenomenon in nature to regulate, for example, the activity of proteins ${ }^{6}$, especially of enzymes. The synthesis and biological investigation, however, of dimeric structures derived from triterpenes have been fairly studied ${ }^{7-10}$. The chemistry of triterpenoic dimers started with Dolby's and Debono's dimerization ${ }^{11}$ of citronellal. Since then several dimers have been described ${ }^{7}$. Of special interest are 3-amino-oleanolic acid derived dimers holding an adipinic acid-derived spacer ${ }^{12}$ showing some activity for the HIV-1 protease; oleanolic acid derivatives 13,14 were inhibitors of the glycogen phosphorylase.

The number of dimers obtained with an incorporation of the C-28 carboxyl group remains limited probably due to its steric hindrance ${ }^{15}$ also found for lupane derived dimers ${ }^{16}$. Only a few dimers holding an amide spacer ${ }^{13,14,16}$ have been described so far. These compounds were tested as enzyme inhibitors or antivirals but - by and large - no cytotoxic data have been provided for these compounds.

*Corresponding author: René Csuk

Email address: rene.csuk@chemie.uni-halle.de DOI: http://dx.doi.org/10.13171/mjc91190811415rc
To surpass some problems usually associated with low solubility and to develop an application providing a slow-release of the drug, trans-dermal applications have been suggested and used quite successfully for different kinds of drugs ${ }^{17}$. Thereby, the transdermal application involves the application of ointments, creams or gels to the skin, and the active ingredients of which are absorbed by the skin. This allows them to enter the bloodstream and from there to their actual site of action. Transdermal patches, also known as "transdermal therapeutic systems" (TTS), are a frequently used method of transdermal application. They can release drug substances slowly and in a controlled manner from a drug depot. As a prerequisite for their use, these substances must not exert any unfavorable side-effects, such as cytotoxicity.

\section{Results and Discussion}

Oleanolic acid (OA) and ursolic acid (UA) exert weak cytotoxicity; previous studies showed an increase in cytotoxicity upon their acetylation ${ }^{18-20}$. Also, OA and UA derived amides holding an additional amino group attached distally to an alkyl group (or a cyclic amine) were shown to be higher cytotoxic than their parent compounds ${ }^{18-23}$.

We became interested in the syntheses of dimeric OA and UA amides linked together by an alkyl chain of variable length. OA and UA were acetylated and 3-O-acetylated $\mathbf{1}^{24-26}$ and $\mathbf{2}^{27,28}$ were obtained (Scheme 1). The reaction of $\mathbf{1}$ with oxalyl chloride followed by the addition of 0.5 equivalents of ethylene-diamine gave dimeric 3. 
The reaction of $\mathbf{1}$ with various diamines of variable spacer length furnished OA derived dimers 3-8. Similarly, from 2, UA derived dimers 9-14 were obtained in $56-88 \%$ isolated yield. Deacetylation of
3-14 gave dimers 15-26, each holding an unprotected hydroxyl moiety at positions C-3 and C-3', respectively.

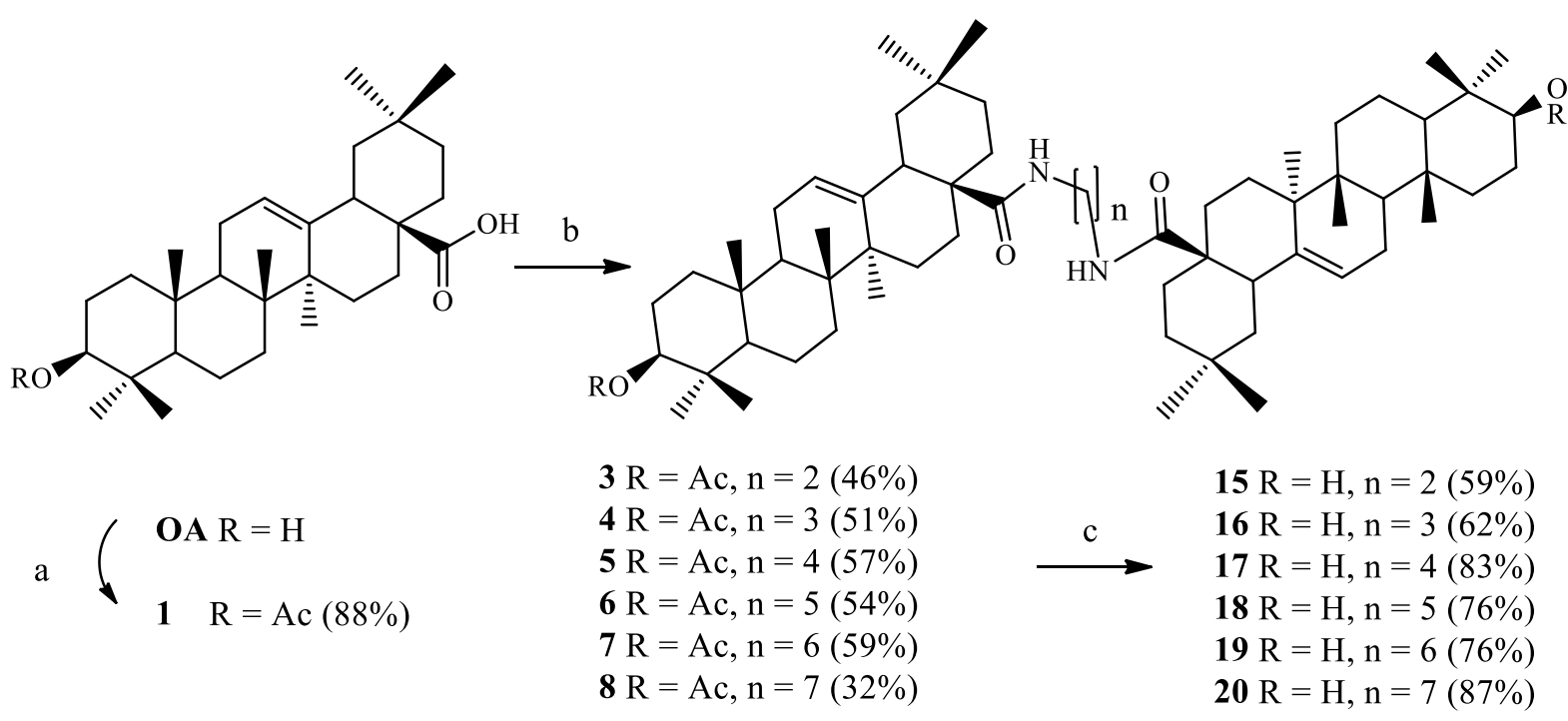

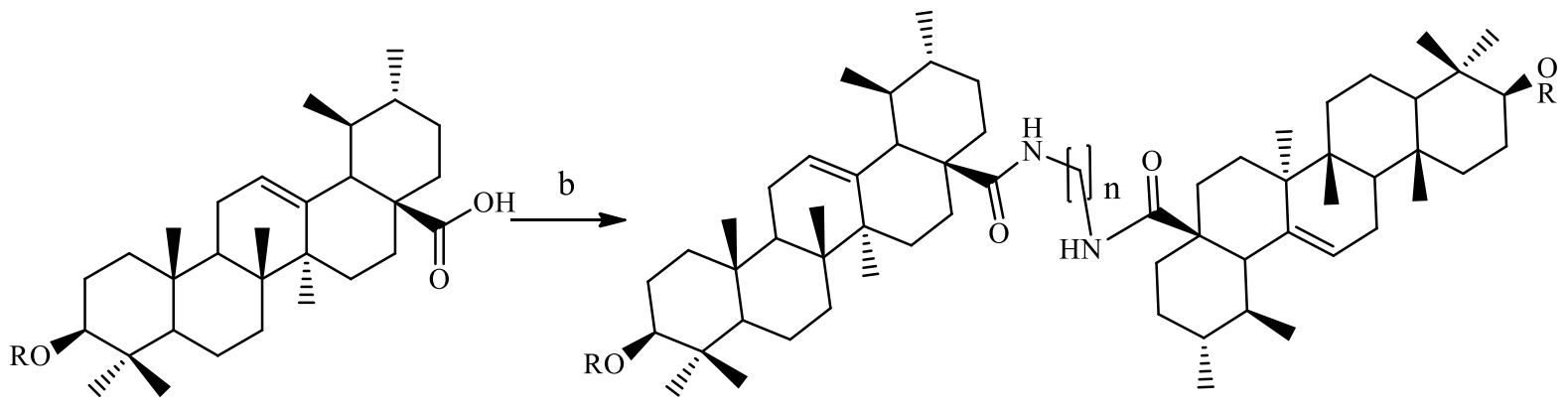<smiles></smiles>

$$
\begin{aligned}
9 \mathrm{R} & =\mathrm{Ac}, \mathrm{n}=2(73 \%) \\
10 \mathrm{R} & =\mathrm{Ac}, \mathrm{n}=3(88 \%) \\
11 \mathrm{R} & =\mathrm{Ac}, \mathrm{n}=4(87 \%) \\
12 \mathrm{R} & =\mathrm{Ac}, \mathrm{n}=5(81 \%) \\
13 \mathrm{R} & =\mathrm{Ac}, \mathrm{n}=6(85 \%) \\
14 \mathrm{R} & =\mathrm{Ac}, \mathrm{n}=7(56 \%)
\end{aligned}
$$$$
21 \mathrm{R}=\mathrm{H}, \mathrm{n}=2(85 \%)
$$

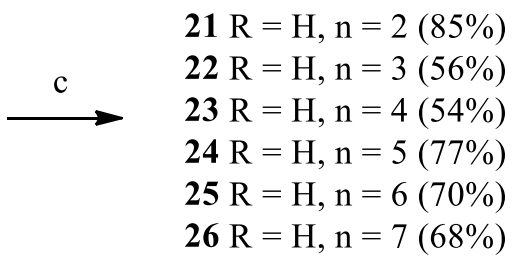

$21 \mathrm{R}=\mathrm{H}, \mathrm{n}=2(85 \%)$

$23 \mathrm{R}=\mathrm{H}, \mathrm{n}=4(54 \%)$

$\mathrm{R}=\mathrm{H}, \mathrm{n}=5(77 \%)$

$26 \mathrm{R}=\mathrm{H}, \mathrm{n}=7(68 \%)$

Scheme 1. a) Acetic anhydride, pyridine, cat. DMAP, stirring at $25^{\circ} \mathrm{C}, 1$ day; b) i. oxalyl chloriode, triethylamine, dichloromethane, cat. DMF, stirring at $25^{\circ} \mathrm{C}, 2 \mathrm{~h}$, ii. DCM, 1,n-diamine, stirring at $25^{\circ} \mathrm{C}, 2 \mathrm{~h}$; c) $\mathrm{KOH}, \mathrm{MeOH}$, microwaves, $\lambda=365 \mathrm{~nm}, 100^{\circ} \mathrm{C}, 5 \mathrm{~min}$.

All of the dimers were screened for their cytotoxic activity in sulforhodamine assays (SRB), but none of these compounds showed any significant cytotoxicity ( $\mathrm{EC}_{50}>30 \square \mathrm{M}$ cut-off) when tested with several human tumor cell lines (A375, HT29, SW1736, MCF7, A2780, FaDu, A549) as well with nonmalignant mouse fibroblasts (NIH 3T3). No cytotoxic effect was observed even upon applying a prolonged incubation time of 96 hours. Presently, the skin penetration and the stability of these dimers as well as they're in vivo degradation is studied in more detail in our laboratories.

\section{Conclusion}

A slow-release form of ursolic and oleanolic acid, amide-spacered dimers were synthesized from the parent acids and diamines of variable chain lengths. These dimers were assayed in SRB assays; even upon long term incubation up to 96 hours, none of these compounds showed any significant cytotoxicity in sulforhodamine B assays.

\section{Experimental}

NMR spectra were recorded using the Varian spectrometers Gemini 2000 or Unity 500 ( $\square$ given in ppm, $J$ in $\mathrm{Hz}$; typical experiments: $\mathrm{H}-\mathrm{H}-\mathrm{COSY}$, HMBC, HSQC, NOESY; Fig. 1 shows the numbering scheme as well as key HMBC correlations), MS 
spectra were taken on a Finnigan MAT LCQ 7000 (electrospray, voltage $4.1 \mathrm{kV}$, sheath gas nitrogen) instrument. The optical rotations were measured on a Perkin-Elmer polarimeter at $20{ }^{\circ} \mathrm{C}$; TLC was performed on silica gel (Merck 5554, detection with cerium molybdate reagent); melting points are uncorrected (Leica hot stage microscope or BÜCHI Melting Point M-565), and elemental analyses were performed on a Foss-Heraeus Vario EL (CHNS) unit.
IR spectra were recorded on a Perkin Elmer FT-IR spectrometer Spectrum 1000 or a Perkin-Elmer Spectrum Two (UATR Two Unit). The solvents were dried according to usual procedures. The purity of the compounds was determined by HPLC and found to be $>96 \%$. Ursolic (UA), oleanolic (OA) acid were obtained from different commercial suppliers in bulk quantities. All compounds were obtained as colorless solids.
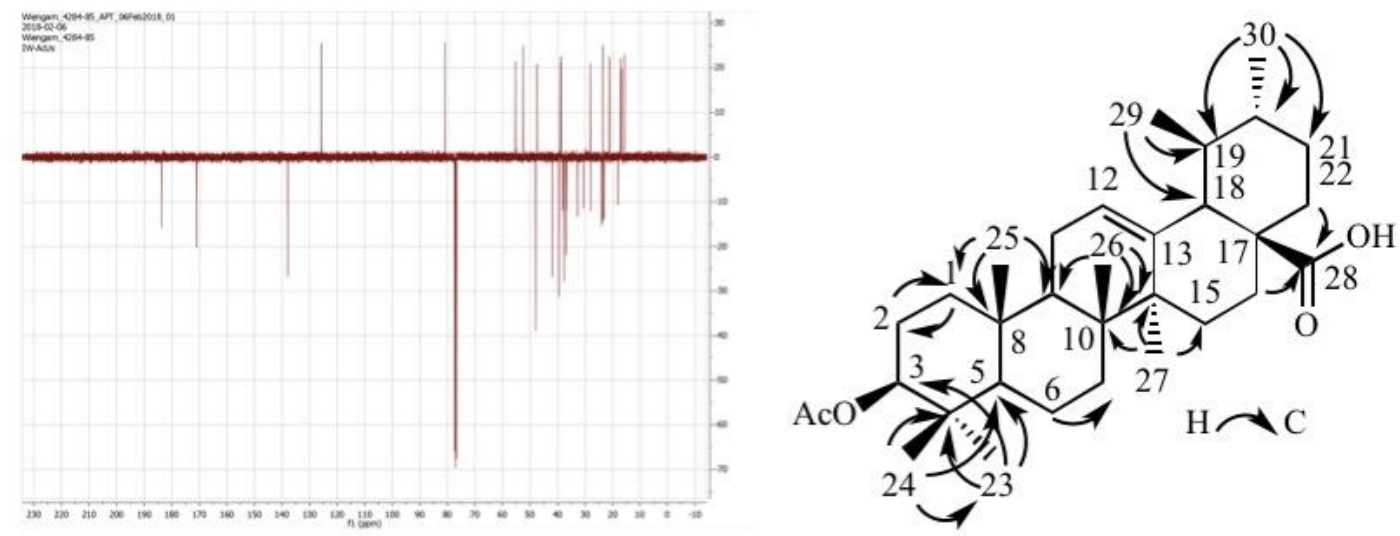

Figure 1. A typical ${ }^{13} \mathrm{C}$ APT-NMR spectrum of 2, numbering scheme and key HMBC correlations.

(3ß) 3-Acetyloxy-olean-12-en-28-oic acid (1) Acetylation of $\mathbf{O A}(5.0 \mathrm{~g}, 0.01 \mathrm{~mol})$ with acetic anhydride $(3.11 \mathrm{~mL}, 0.03 \mathrm{~mol})$ in dry pyridine $(50$ $\mathrm{mL}$ ) in the presence of a catal. amount of DMAP for 1 day at $25{ }^{\circ} \mathrm{C}$ followed by usual aqueous work-up and column chromatography (silica gel, $n$-hexane/ethyl acetate, 8:2) gave $\mathbf{1}\left(4.4 \mathrm{~g}, 88 ; \mathrm{R}_{F}=\right.$ 0.7 (silica gel, toluene/ethyl acetate/formic acid/heptane, 80:26:5:1); m.p.: 263-265 ${ }^{\circ} \mathrm{C}$ (lit..$^{29}$ 260-261 $\left.{ }^{\circ} \mathrm{C}\right) ;[\alpha]_{\mathrm{D}}=+117.7^{\circ}\left(c 0.37, \mathrm{CHCl}_{3}\right)$, (lit.: ${ }^{30}$ $+119^{\circ}\left(c 0.1, \mathrm{CHCl}_{3}\right)$; MS (ESI, MeOH): $\mathrm{m} / \mathrm{z}=497.5$ $\left(75 \%,[\mathrm{M}-\mathrm{H}]^{-}\right), 995.2\left(100 \%,[2 \mathrm{M}-\mathrm{H}]^{-}\right), 1017.7$ $\left(29 \%,[2 \mathrm{M}-2 \mathrm{H}+\mathrm{Na}]^{-}\right)$.

(3ß) 3-Acetyloxy-urs-12-en-28-oic acid (2)

Following the procedure given for the synthesis of $\mathbf{1}$, from UA compound 2 (3.5 g, 64\%) was obtained; $\mathrm{R}_{F}=0.7$ (silica gel, toluene/ethyl acetate/formic acid/heptane, 80:26:5:1); m.p.: $242-244^{\circ} \mathrm{C}$ (lit.: ${ }^{31}$ $\left.242.7-244 .{ }^{\circ} \mathrm{C}\right) ;[\alpha]_{\mathrm{D}}=+69.89^{\circ}\left(c 0.86, \mathrm{CHCl}_{3}\right)$, (lit.: ${ }^{31}+71.2^{\circ}$ (c 1.0, $\left.\mathrm{CHCl}_{3}\right)$; $\mathrm{MS}$ (ESI, $\left.\mathrm{MeOH}\right)$ : $m / z=497.5\left(64 \%,[\mathrm{M}-\mathrm{H}]^{-}\right), 542.9\left(30 \%,\left[\mathrm{M}+\mathrm{HCO}_{2}\right]^{-}\right.$ )$, \quad 995.1\left(68 \%,[2 \mathrm{M}-\mathrm{H}]^{-}\right), \quad 1017.5(100 \%$, $\left.[2 \mathrm{M}-2 \mathrm{H}+\mathrm{Na}]^{-}\right)$.

\section{General procedure for the synthesis of the dimers} To a solution of 1 or $2(0.37 \mathrm{mmol})$ in dry DCM (abs., $30 \mathrm{~mL})$ at $0{ }^{\circ} \mathrm{C}$ oxalyl chloride $(1.2 \mathrm{mmol})$, triethylamine $(1.2 \mathrm{mmol})$ and $\mathrm{DMF}$ (2 drops) were slowly added, and the mixture was allowed to react at $25{ }^{\circ} \mathrm{C}$ for $2 \mathrm{~h}$. The volatiles were removed under reduced pressure, the residue was dissolved in dry $\operatorname{DCM}(5 \mathrm{~mL})$, and the amine $(0.18 \mathrm{mmol})$ and catalyst amounts of DMAP were added. After stirring at $25^{\circ} \mathrm{C}$ for $1 \mathrm{~h}$, usual aqueous workup followed by column chromatography gave dimeric 3-14.

\section{General procedure for deacetylation}

To a solution of compound 3-14, (1 equiv) in dry $\mathrm{MeOH}(5 \mathrm{~mL})$ powdered $\mathrm{KOH}$ (20 equiv) was added, and the mixture was stirred at $100{ }^{\circ} \mathrm{C}$ (microwave irradiation, $\square=365 \mathrm{~nm}$ ) for $5 \mathrm{~min}$. Usual aqueous workup followed by column chromatography furnished products 15-26; respectively.

\section{$\mathbf{N}^{1}, \quad \mathbf{N}^{2}$-Bis-[(3ß)-3-acetyloxy-olean-12-en-28-oyl]-} 1,2-diaminoethane (3)

Compound 3 (0.17 g, 46\%) showed: m.p. $240-242^{\circ} \mathrm{C}$; $\mathrm{R}_{F}=0.7$ (silica gel, toluene/ethyl acetate/formic $\mathrm{acid} /$ heptane, 80:26:5:1); $[\alpha]_{\mathrm{D}}=+49.56^{\circ}$ (c 0.32 , $\left.\mathrm{CHCl}_{3}\right)$;

IR (KBr): $v=3424 b r, 2948 m, 2878 m, 1736 s, 1638 m$, $1524 m, 1466 m, 1368 m, 1246 m, 1212 w, 1186 w$, $1148 w, 1096 w, 1028 m, 1008 m, 986 \mathrm{~cm}^{-1}$;

${ }^{1} \mathrm{H} \mathrm{NMR}\left(500 \mathrm{MHz}, \mathrm{CDCl}_{3}\right): \delta=6.47(s, 2 \mathrm{H}, 2 \times \mathrm{NH})$, $5.39(t, J=3.5 \mathrm{~Hz}, 2 \mathrm{H}, 2 \times 12-\mathrm{H}), 4.52-4.46(m, 2 \mathrm{H}$, $2 \times 3-\mathrm{H}), 3.50-3.40(m, 2 \mathrm{H}, 2 \times 33-\mathrm{H}), 3.11(t d, 2 \mathrm{H}$, $2 \times 33-\mathrm{H}), 2.54(d d, J=13.1,4.0 \mathrm{~Hz}, 2 \mathrm{H}, 2 \times 18-\mathrm{H})$, $2.04(s, 6 \mathrm{H}, 2 \times 32-\mathrm{H}), 2.00(d d, J=13.8 \mathrm{~Hz}, 4.0 \mathrm{~Hz}$, $2 \mathrm{H}, 2 \times 16-\mathrm{H}), 1.96-1.89(\mathrm{~m}, 2 \mathrm{H}, 2 \times 11-\mathrm{H})$, $1.72-1.55(m, 18 \mathrm{H}, 2 \times 1-\mathrm{H}+2 \times 2-\mathrm{H}+2 \times 6-\mathrm{H}+$ $2 \times 7-\mathrm{H}+2 \times 9-\mathrm{H}+2 \times 11-\mathrm{H}+2 \times 16-\mathrm{H}+2 \times 19-\mathrm{H})$, $1.53-1.49(m, 2 \mathrm{H}, 2 \times 15-\mathrm{H}), 1.47-1.40(m, 2 \mathrm{H}, 2 \times$ $22-\mathrm{H}), 1.40-1.26(d d, J=12.5,3.3 \mathrm{~Hz}, 6 \mathrm{H}, 2 \times 6-\mathrm{H}+$ $2 \times 7-\mathrm{H}+2 \times 21-\mathrm{H}), 1.25(d d, J=10.1,2.2 \mathrm{~Hz}, 2 \mathrm{H}$, $2 \times 22-\mathrm{H}), 1.19(d, J=3.9 \mathrm{~Hz}, 4 \mathrm{H}, 2 \times 19-\mathrm{H}+2 \times 21-$ $\mathrm{H}), 1.15(s, 6 \mathrm{H}, 2 \times 27-\mathrm{H}) 1.02(d d, J=10.6,3.3 \mathrm{~Hz}$, $4 \mathrm{H}, 2 \times 1-\mathrm{H}+2 \times 15-\mathrm{H}), 0.93(s, 6 \mathrm{H}, 2 \times 25-\mathrm{H}), 0.90$ $(s, 6 \mathrm{H}, 2 \times 29-\mathrm{H}), 0.89(s, 6 \mathrm{H}, 2 \times 30-\mathrm{H}), 0.86(s, 6 \mathrm{H}$, 
$2 \times 23-\mathrm{H}), 0.85(s, 6 \mathrm{H}, 2 \times 26-\mathrm{H}), 0.82(s, 2 \mathrm{H}, 2 \times 5-$ $\mathrm{H}), 0.74(s, 6 \mathrm{H}, 2 \times 24-\mathrm{H})$;

${ }^{13} \mathrm{C}$ NMR $\left(125 \mathrm{MHz}, \mathrm{CDCl}_{3}\right): \delta=179.3(2 \times \mathrm{C}-28)$, 170.9 ( 2 x C-28), 144.5 ( 2 x C-13), 122.9 ( 2 x C-12), 80.8 ( 2 x C-3), 55.19 ( 2 x C-5), 47.5 ( 2 x C-9), 46.6 ( 2 x C-17), 46.2 (2 x C-19), 41.9 ( 2 x C-18), $41.9(2 \times$ C-14), 39.9 (2 x C-33), 39.4 ( 2 x C-8), 38.1 ( $2 \times$ C-1), 37.6 ( 2 x C-4), 36.8 ( $2 \times$ C-10), 34.1 ( $2 \times$ C-21), 32.9 (2 x C-29), 32.8 (2 x C-7), 32.2 (2 x C-22), 30.6 ( 2 x C-20), 28.0 ( $2 \times$ C-23), 27.3 ( $2 \times$ C-15), 25.7 ( $2 \times$ C27), 23.6 ( $2 \times \mathrm{C}-11), 23.5$ ( $2 \times \mathrm{C}-30), 23.5$ ( $2 \times \mathrm{C}-2)$, 23.5 ( 2 x C-16), 21.2 ( 2 x C-32), 18.1 ( $2 \times$ C-6), 16.8 (2 x C-24), 16.6 (2 x C-26), 15.4 (2 x C-25) ppm; MS (ESI, MeOH): $m / z=1021.7\left(50 \%,[\mathrm{M}+\mathrm{H}]^{+}\right)$, $1043.8\left(100 \%,[\mathrm{M}+\mathrm{Na}]^{+}\right)$;

analysis calcd for $\mathrm{C}_{66} \mathrm{H}_{104} \mathrm{~N}_{2} \mathrm{O}_{6}$ (1021.54): C 77.60, H 10.26, N 2.74; found: C 77.41, H 10.45, N 2.49.

$\mathbf{N}^{1}, \quad \mathbf{N}^{3}$-Bis-[(3ß)-3-acetyloxy-olean-12-en-28-oyl]1,3-diaminopropane (4)

Compound 4 (0.18g, 51\%) showed: m.p. $204-206^{\circ} \mathrm{C}$; $\mathrm{R}_{F}=0.5$ (silica gel, toluene/ethyl acetate/formic $\mathrm{acid} /$ heptane, 80:26:5:1); $[\alpha]_{\mathrm{D}}=+52.27^{\circ}$ ( $c 0.32$, $\left.\mathrm{CHCl}_{3}\right)$;

IR (KBr): $v=3337 b r, 2945 m, 2863 w, 1736 m, 1635 m$, $1526 m, 1433 m, 1365 m, 1244 s, 1026 \mathrm{~m} \mathrm{~cm}^{-1}$;

${ }^{1} \mathrm{H} \mathrm{NMR}\left(500 \mathrm{MHz}, \mathrm{CDCl}_{3}\right): \delta=6.42(t, J=5.9 \mathrm{~Hz}$, $2 \mathrm{H}, 2 \times \mathrm{NH}), 5.40(t, J=3.3 \mathrm{~Hz}, 2 \mathrm{H}, 2 \times 12-\mathrm{H}), 4.48$ $(d d, J=10.0,5.9 \mathrm{~Hz}, 2 \mathrm{H}, 2 \times 3-\mathrm{H}), 3.25(d q, J=12.9$, $6.5 \mathrm{~Hz}, 2 \mathrm{H}, 2 \mathrm{x} 33-\mathrm{H}), 3.11(d q, J=12.3,6.0 \mathrm{~Hz}, 2 \mathrm{H}$, $2 \times 33-\mathrm{H}), 2.64(d d, J=13.0,3.1 \mathrm{~Hz}, 2 \mathrm{H}, 2 \times 18-\mathrm{H})$, $2.04(s, 6 \mathrm{H}, 2 \times 32-\mathrm{H}), 1.97(t d, J=13.6,3.5 \mathrm{~Hz}, 2 \mathrm{H}$, $2 \times 16-\mathrm{H}), 1.92-1.87(m, 2 \mathrm{H}, 2 \times 11-\mathrm{H}), 1.78-1.55(m$, $20 \mathrm{H}, 2 \times 1-\mathrm{H}+2 \times 2-\mathrm{H}+2 \times 6-\mathrm{H}+2 \times 7-\mathrm{H}+2 \times 9-$ $\mathrm{H}+2 \times 11-\mathrm{H}+2 \times 16-\mathrm{H}+2 \times 19-\mathrm{H}+2 \times 34-\mathrm{H})$, $1.52-1.49(m, 2 \mathrm{H}, 2 \times 15-\mathrm{H}), 1.44(d d, J=12.6,3.5$ $\mathrm{Hz}, 2 \mathrm{H}, 2 \mathrm{x} 22-\mathrm{H}), 1.35(q d, J=14.0,13.0,3.5 \mathrm{~Hz}$, $6 \mathrm{H}, 2 \times 6-\mathrm{H}+2 \times 7-\mathrm{H}+2 \times 21-\mathrm{H}), 1.26(d, J=13.1$ $\mathrm{Hz}, 2 \mathrm{H}, 2$ x 22-H), 1.21-1.15 (m, 4H, 2 x 19-H + 2 x $21-\mathrm{H}), 1.14(s, 6 \mathrm{H}, 2 \times 27-\mathrm{H}), 1.06-1.00(m, 4 \mathrm{H}, 2 \times$ $1-\mathrm{H}+2 \times 15-\mathrm{H}), 0.92(s, 6 \mathrm{H}, 2 \times 25-\mathrm{H}), 0.91(s, 6 \mathrm{H}$, $2 \times 29-\mathrm{H}), 0.90(s, 6 \mathrm{H}, 2 \times 30-\mathrm{H}), 0.86(s, 6 \mathrm{H}, 2 \times$ $23-\mathrm{H}), 0.84(s, 6 \mathrm{H}, 2 \times 26-\mathrm{H}), 0.82(s, 2 \mathrm{H}, 2 \times 5-\mathrm{H})$, $0.74(s, 6 \mathrm{H}, 2 \times 24-\mathrm{H}) \mathrm{ppm}$;

${ }^{13} \mathrm{C}$ NMR $\left(125 \mathrm{MHz}, \mathrm{CDCl}_{3}\right): \delta=178.5(2 \times \mathrm{C}-28)$, 170.9 ( $2 \times \mathrm{C}-28), 144.6$ ( $2 \times \mathrm{C}-13), 122.6(2 \times \mathrm{C}-12)$, 80.8 ( 2 x C-3), 55.1 ( $2 \times$ C-5), 47.4 ( 2 x C-9), 46.6 (2 x C-17), 46.2 (2 x C-19), 41.95 (2 x C-18), 41.8 (2 x C-14), 39.3 (2 x C-8), 38.1 ( 2 x C-1), 37.6 ( $2 \times \mathrm{C}-4), 36.8$ ( $2 \times \mathrm{C}-10), 36.0$ ( $2 \times \mathrm{C}-33), 34.2$ (2 x C-21), 33.0 (2 x C-29), 32.9 (2 x C-7), 32.3 ( 2 x C-22), 30.7 (2 x C-20), 29.7 (C-34), 28.0 (2 x C-23), 27.3 ( 2 x C-15), 25.7 ( 2 x C-27), 23.6 ( $2 \times$ C-11), 23.6 (2 x C-30), 23.5 (2 x C-2), 23.4 (2 x C-16), 21.2 ( 2 x C-32), 18.1 ( $2 \times \mathrm{C}-6), 16.9$ ( $2 \times \mathrm{C}-24), 16.6$ ( $2 \times$ C-26), 15.4 (2 x C-25) ppm;

MS (ESI, MeOH): $m / z=1035.7\left(86 \%,[\mathrm{M}+\mathrm{H}]^{+}\right)$, $1057.8\left(100 \%[\mathrm{M}+\mathrm{Na}]^{+}\right.$;

analysis calcd for $\mathrm{C}_{67} \mathrm{H}_{106} \mathrm{~N}_{2} \mathrm{O}_{6}$ (1035.57): C 77.71, H 10.32, N 2.71; found: C 77.50, H 10.46, N 2.42.
$\mathbf{N}^{1}, \quad \mathbf{N}^{4}$-Bis-[(3ß)-3-acetyloxy-olean-12-en-28-oyl]1,4-diaminobutane (5)

Compound 5 (0.21g, 57\%) showed: m.p. $180-186^{\circ} \mathrm{C}$; $\mathrm{R}_{F}=0.5$ (silica gel, toluene/ethyl acetate/formic $\mathrm{acid} /$ heptane, 80:26:5:1); $[\alpha]_{\mathrm{D}}=+46.94^{\circ}$ (c 0.37 , $\left.\mathrm{CHCl}_{3}\right)$;

IR $(\mathrm{KBr}): v=3398 b r, 2950 m, 2874 m, 1736 s, 1644 m$, $1522 m, 1466 m, 1366 w, 1246 s, 1212 w, 1148 w, 1048 w$, $1098 m, 1006 w \mathrm{~cm}^{-1}$;

${ }^{1} \mathrm{H} \mathrm{NMR}\left(500 \mathrm{MHz}, \mathrm{CDCl}_{3}\right): \delta=6.04(s, 2 \mathrm{H}, 2 \times \mathrm{NH})$, $5.38(t, J=3.6 \mathrm{~Hz}, 2 \mathrm{H}, 2 \times 12-\mathrm{H}), 4.52-4.47(\mathrm{~m}, 2 \mathrm{H}$, $2 \times 3-\mathrm{H}), 3.36(d q, J=13.0,6.6 \mathrm{~Hz}, 2 \mathrm{H}, 2 \times 33-\mathrm{H})$, $3.04(d q, J=11.3,6.3 \mathrm{~Hz}, 2 \mathrm{H}, 2 \times 33-\mathrm{H}), 2.54(d d, J$ $=13.0,4.0 \mathrm{~Hz}, 2 \mathrm{H}, 2 \times 18-\mathrm{H}), 2.06(s, 6 \mathrm{H}, 2 \times 32-\mathrm{H})$, $1.97(d, J=3.6 \mathrm{~Hz}, 2 \mathrm{H} 2 \times 16-\mathrm{H}), 1.92(d d, J=8.8$, $3.2 \mathrm{~Hz}, 2 \mathrm{H}, 2 \times 11-\mathrm{H}), 1.81-1.51(m, 22 \mathrm{H}, 2 \times 1-\mathrm{H}+$ $2 \times 2-\mathrm{H}+2 \times 6-\mathrm{H}+2 \times 7-\mathrm{H}+2 \times 9-\mathrm{H}+2 \times 11-\mathrm{H}+$ $2 \times 16-\mathrm{H}+2 \times 19-\mathrm{H}+2 \times 34-\mathrm{H}), 1.48(d t, J=6.5$, $3.4 \mathrm{~Hz}, 2 \mathrm{H}, 2 \times 15-\mathrm{H}), 1.43(s, 2 \mathrm{H}, 2 \times 22-\mathrm{H})$, $1.41-1.35(m, 6 \mathrm{H}, 2 \times 6-\mathrm{H}+2 \times 7-\mathrm{H}+2 \times 21-\mathrm{H})$, $1.30-1.25(m, 2 \mathrm{H} 2 \times 22-\mathrm{H}), 1.23-1.19(m, 4 \mathrm{H}$, $2 \times 19-\mathrm{H}+2 \times 21-\mathrm{H}), 1.16(s, 6 \mathrm{H} 2 \times 27-\mathrm{H}), 1.06$ (s, 4H, $2 \times 1-\mathrm{H}+2 \times 15-\mathrm{H}), 0.97(\mathrm{~d}, J=6.6 \mathrm{~Hz}, 6 \mathrm{H}$, $2 \times 25-\mathrm{H}), 0.95(s, 6 \mathrm{H} 2 \times 29-\mathrm{H}), 0.91(s, 6 \mathrm{H} 2 \times$ $30-\mathrm{H}), 0.88(s, 6 \mathrm{H}, 2 \times 23-\mathrm{H}), 0.87(s, 6 \mathrm{H} 2 \times 26-\mathrm{H})$, $0.86-0.82(m, 2 \mathrm{H}, 2 \times 5-\mathrm{H}), 0.76(s, 6 \mathrm{H} 2 \times 24-\mathrm{H})$ ppm;

${ }^{13} \mathrm{C}$ NMR (125 MHz, $\left.\mathrm{CDCl}_{3}\right): \delta=178.2(2 \times \mathrm{C}-28)$, 170.9 ( 2 x C-28), 145.0 ( 2 x C-13), 122.6 ( $2 \times$ C-12), 80.8 ( 2 x C-3), 55.1 ( 2 x C-5), 47.4 (2 x C-9), 46.7 (2 x C-17), 46.2 (2 x C-19), 42.2 ( 2 x C-18), 42.0 (2 x C-14), 39.3 ( $2 \times \mathrm{C}-8), 38.9$ (2 x C-33), 38.1 (2 x C-1), 37.6 (2 x C-4), 36.8 (2 x C-10), 34.1 (2 x C-21), 32.9 ( $2 \times \mathrm{C}-29), 32.6$ (2 x C-7), 32.3 ( 2 x C-22), 30.7 ( 2 x C-20), 28.0 ( 2 x C-23), 27.2 ( $2 \times \mathrm{C}-15), 26.9$ ( $2 \times \mathrm{C}-34), 25.6$ ( $2 \times \mathrm{C}-27), 23.7$ (2 x C-11), 23.6 (2 x C-30), 23.5 (2 x C-2), 23.4 (2 x C-16), 21.2 (2 x C-32), 18.1 (2 x C-6), 16.9 ( 2 x C-24), 16.6 ( 2 x C-26), 15.4 ( 2 x C-25) ppm;

MS (ESI, MeOH): $m / z=1049.7\left(78 \%,[\mathrm{M}+\mathrm{H}]^{+}\right)$, $1071.7\left(100 \%,[\mathrm{M}+\mathrm{Na}]^{+}\right)$;

analysis calcd for $\mathrm{C}_{68} \mathrm{H}_{108} \mathrm{~N}_{2} \mathrm{O}_{6}$ (1049.60): C 77.81, H 10.37, N 2.67; found: C 77.62, H 10.53, N 2.55.

$\mathbf{N}^{1}, \quad \mathbf{N}^{5}$-Bis-[(3ß)-3-acetyloxy-olean-12-en-28-oyl]1,5-diaminopentane (6)

Compound $6(0.21 \mathrm{~g}, 54 \%)$ showed: m.p. $175^{\circ} \mathrm{C}$; $\mathrm{R}_{F}=0.5$ (silica gel, toluene/ethyl acetate/formic $\mathrm{acid} /$ heptane, 80:26:5:1); $[\alpha]_{\mathrm{D}}=+47.07^{\circ}($ c 0.333 , $\left.\mathrm{CHCl}_{3}\right)$;

IR (KBr): $v=3426 b r, 2946 s, 2876 m, 1735 s, 1641 m$, $1522 m, 14465 m, 1366 m, 1245 s, 1212 w, 1148 w$, $1096 w, 1027 m, 1006 m, 986 m, 970 w \mathrm{~cm}^{-1}$;

${ }^{1} \mathrm{H} \mathrm{NMR}\left(500 \mathrm{MHz}, \mathrm{CDCl}_{3}\right): \delta=5.91(s, 2 \mathrm{H}, 2 \times \mathrm{NH})$, $5.37(t, J=3.6 \mathrm{~Hz}, 2 \mathrm{H}, 2 \times 12-\mathrm{H}), 4.49(d d, J=10.3$, $5.7 \mathrm{~Hz}, 2 \mathrm{H}, 2 \times 3-\mathrm{H}), 3.34(d q, J=13.8,7.1 \mathrm{~Hz}, 2 \mathrm{H}$, $2 \times 33-\mathrm{H}), 3.03-2.93(m, 2 \mathrm{H}, 2 \times 33-\mathrm{H}), 2.50(d d, J=$ 13.2, $4.0 \mathrm{~Hz}, 2 \mathrm{H}, 2 \times 18-\mathrm{H}), 2.05(s, 6 \mathrm{H}, 2 \times 32-\mathrm{H})$, $1.98(d, J=3.8 \mathrm{~Hz}, 2 \mathrm{H}, 2 \times 16-\mathrm{H}), 1.92(d t, J=8.8$, $4.8 \mathrm{~Hz}, 2 \mathrm{H}, 2$ x 11-H), 1.81-1.51 (m, 26H, 2 × 1-H + $2 \times 2-\mathrm{H}+2 \times 6-\mathrm{H}+2 \times 7-\mathrm{H}+2 \times 9-\mathrm{H}+2 \times 11-\mathrm{H}+$ 
$2 \times 16-\mathrm{H}+2 \times 19-\mathrm{H}+2 \times 34-\mathrm{H}+2 \times 35-\mathrm{H}), 1.48$ $(q, J=7.0,6.1 \mathrm{~Hz}, 2 \mathrm{H}, 2 \times 15-\mathrm{H}), 1.45-1.40(m, 2 \mathrm{H}$, $2 \times 22-\mathrm{H}), 1.36(d d d, J=17.6,8.2,4.3 \mathrm{~Hz}, 6 \mathrm{H}, 2 \times$ $6-\mathrm{H}+2 \times 7-\mathrm{H}+2 \times 21-\mathrm{H}), 1.33-1.24(m, 2 \mathrm{H}, 2 \times$ 22-H), 1.22-1.17 (m, 4H, 2 x 19-H + 2 x 21-H), 1.16 $(s, 6 \mathrm{H}, 2 \times 27-\mathrm{H}), 1.12-1.00(m, 4 \mathrm{H}, 2 \times 1-\mathrm{H}+2 \times$ $15-\mathrm{H}), 0.94(s, 6 \mathrm{H}, 2 \times 25-\mathrm{H}), 0.90(s, 6 \mathrm{H}, 2 \times 29-\mathrm{H})$, $0.90(s, 6 \mathrm{H}, 2 \times 30-\mathrm{H}), 0.87(s, 6 \mathrm{H}, 2 \times 23-\mathrm{H}), 0.86$ $(s, 6 \mathrm{H}, 2 \times 26-\mathrm{H}), 0.83(m, 2 \mathrm{H}, 2 \times 5-\mathrm{H}), 0.76(s, 6 \mathrm{H}$, 2 x 24-H) ppm;

${ }^{13} \mathrm{C}$ NMR (125 MHz, $\left.\mathrm{CDCl}_{3}\right): \delta=178.2(2 \times \mathrm{C}-28)$, 170.9 ( 2 x C-28), 145.1 ( 2 x C-13), 122.6 (2 x C-12), 80.7 ( 2 x C-3), 55.1 ( 2 x C-5), 47.4 ( 2 x C-9), 46.7 (2 x C-17), 46.2 (2 x C-19), 42.3 (2 x C-18), 42.0 ( 2 x C-14), 39.3 ( $2 \times$ C-8), 39.3 ( $2 \times$ C-33), 38.1 ( $2 \times$ C-1), $37.6(2 \times$ C-4), 36.8 ( $2 \times$ C-10), 34.1 ( $2 \times$ C-21), 32.9 ( 2 x C-29), 32.5 ( 2 x C-7), 32.3 ( 2 x C-22), 30.7 ( 2 x C-20), 29.1 ( $2 \times \mathrm{C}-34), 28.0$ ( $2 \times \mathrm{C}-23), 27.2$ ( $2 \times$ C-27), 25.6 ( $2 \times \mathrm{C}-15), 24.6$ (C-35), 23.7 ( $2 \times \mathrm{C}-11)$, 23.6 ( $2 \times$ C-30), 23.5 ( $2 \times$ C-2), 23.5 ( $2 \times$ C-16), 21.2 (2 x C-32), 18.1 ( 2 x C-6), 16.9 (2 x C-24), 16.6 ( 2 x C-26), 15.4 (2 x C-25) ppm;

MS (ESI, MeOH): $m / z=1063.7\left(100 \%,[\mathrm{M}+\mathrm{H}]^{+}\right)$, $1085.7\left(80 \%,[\mathrm{M}+\mathrm{Na}]^{+}\right)$;

analysis calcd for $\mathrm{C}_{69} \mathrm{H}_{110} \mathrm{~N}_{2} \mathrm{O}_{6}$ (1063.62): $\mathrm{C} 77.92$, H 10.42, N 2.63; found: C 77.67, H 10.69, N 2.47.

\section{$\mathbf{N}^{1}, \quad \mathbf{N}^{6}$-Bis-[(3ß)-3-acetyloxy-olean-12-en-28-oyl]-} 1,6-diaminohexane (7)

Compound 7 (0.23g, 54\%) showed: m.p. $188-190^{\circ} \mathrm{C}$ (lit.: 13, $14 \quad 191-192{ }^{\circ} \mathrm{C}$ ); $\mathrm{R}_{F}=0.4$ (silica gel, toluene/ethyl acetate/formic acid/heptane, 80:26:5:1); $[\alpha]_{\mathrm{D}}=+47.07^{\circ}\left(c 0.333, \mathrm{CHCl}_{3}\right)$;

IR (KBr): $v=3427 b r, 2946 s, 2876 m, 1736 s, 1654 m$, $1522 m, 1466 m, 1368 m, 1246 s, 1212 w, 1148 w$, $1096 w, 1028 m, 1008 m, 986 m \mathrm{~cm}^{-1}$;

${ }^{1} \mathrm{H} \mathrm{NMR}\left(500 \mathrm{MHz}, \mathrm{CDCl}_{3}\right): \delta=6.41(t, J=6.0 \mathrm{~Hz}$, $2 \mathrm{H}, 2 \times \mathrm{NH}), 5.40(t, J=3.3 \mathrm{~Hz}, 2 \mathrm{H}, 2 \times 12-\mathrm{H}), 4.49$ $(d d, J=9.7,6.2 \mathrm{~Hz}, 2 \mathrm{H}, 2 \times 3-\mathrm{H}), 3.26(d q, J=12.9$, $6.4 \mathrm{~Hz}, 2 \mathrm{H}, 2 \times 33-\mathrm{H}), 3.12(d q, J=12.4,6.0 \mathrm{~Hz}, 2 \mathrm{H}$, $2 \times 33-\mathrm{H}), 2.64(d d, J=13.3,4.6 \mathrm{~Hz}, 2 \mathrm{H}, 2 \times 18-\mathrm{H})$, $2.04(s, 6 \mathrm{H}, 2 \times 32-\mathrm{H}), 1.98(t d, J=13.7,3.7 \mathrm{~Hz}, 2 \mathrm{H}$, $2 \times 16-\mathrm{H}), 1.93-1.87(m, 2 \mathrm{H}, 2 \times 11-\mathrm{H}), 1.79-1.48$ $(m, 30 \mathrm{H}, 2 \times 1-\mathrm{H}+2 \times 2-\mathrm{H}+2 \times 6-\mathrm{H}+2 \times 7-\mathrm{H}+2 \times$ $9-\mathrm{H}+2 \times 11-\mathrm{H}+2 \times 16-\mathrm{H}+2 \times 19-\mathrm{H}+2 \times 34-\mathrm{H}+$ $2 \times 35-\mathrm{H}), 1.46(d, J=2.7 \mathrm{~Hz}, 2 \mathrm{H}, 2 \times 15-\mathrm{H}), 1.43(d$, $J=3.0 \mathrm{~Hz}, 2 \mathrm{H}, 2 \times 22-\mathrm{H}), 1.42-1.33(m, 6 \mathrm{H}, 2 \times 6-\mathrm{H}$ $+2 \times 7-\mathrm{H}+2 \times 21-\mathrm{H}), 1.29(d d, J=18.6,7.7 \mathrm{~Hz}, 2 \mathrm{H}$, $2 \times 22-\mathrm{H}), 1.20(d, J=2.4 \mathrm{~Hz}, 4 \mathrm{H}, 2 \times 19-\mathrm{H}+2 \times$ 21-H), $1.16(s, 6 \mathrm{H}, 2 \times 27-\mathrm{H}), 1.10-0.99(m, 4 \mathrm{H}, 2 \times$ $1-\mathrm{H}+2 \times 15-\mathrm{H}), 0.92(s, 6 \mathrm{H}, 2 \times 25-\mathrm{H}), 0.91(s, 6 \mathrm{H}$ $2 \times 29-\mathrm{H}), 0.90(s, 6 \mathrm{H}, 2 \times 30-\mathrm{H}), 0.86(s, 6 \mathrm{H}, 2 \times$ $23-\mathrm{H}), 0.85(s, 6 \mathrm{H}, 2 \times 26-\mathrm{H}), 0.82(s, 2 \mathrm{H}, 2 \times 5-\mathrm{H})$, $0.74(s, 6 \mathrm{H}, 2 \times 24-\mathrm{H}) \mathrm{ppm}$;

${ }^{13} \mathrm{C}$ NMR $\left(125 \mathrm{MHz}, \mathrm{CDCl}_{3}\right): \delta=178.1$ ( $\left.2 \times \mathrm{C}-28\right)$, 170.9 ( $2 \times \mathrm{C}-28), 145.1$ ( $2 \times \mathrm{C}-13), 122.5$ ( $2 \times \mathrm{C}-12)$, 80.7 ( 2 x C-3), 55.1 ( 2 x C-5), 47.4 (2 x C-9), 46.7 (2 x C-17), 46.2 (2 x C-19), 42.3 (2 x C-18), 42.0 ( 2 x C-14), 39.3 ( 2 x C-8), 39.2 ( 2 x C-33), 38.1 ( $2 \times$ C-1), 37.6 ( 2 x C-4), 36.8 ( 2 x C-10), 34.1 ( $2 \times \mathrm{C}-21), 32.9$ (2 x C-29), 32.5 (2 x C-7), 32.3 (2 x C-22), 30.7 ( 2 x
C-20), 29.2 ( 2 x C-34), 28.0 ( 2 x C-23), 27.2 ( 2 x C27), 26.6 ( $2 \times \mathrm{C}-15), 25.6$ ( $2 \times \mathrm{C}-35), 23.7$ ( $2 \times \mathrm{C}-11)$, 23.6 ( 2 x C-30), 23.5 ( 2 x C-2), 23.4 ( 2 x C-16), 21.2 (2 x C-32), 18.1 ( 2 x C-6), 16.9 ( 2 x C-24), 16.6 ( 2 x C-26), 15.4 (2 x C-25) ppm;

MS (ESI, MeOH): $m / z=558.6\left(5 \%,[\mathrm{M}+\mathrm{Ca}]^{2+}\right)$, $1077.7\left(100 \%,[\mathrm{M}+\mathrm{H}]^{+}\right), 1099.9\left(65 \%,[\mathrm{M}+\mathrm{Na}]^{+}\right)$; analysis calcd for $\mathrm{C}_{70} \mathrm{H}_{112} \mathrm{~N}_{2} \mathrm{O}_{6}$ (1077.65): C 78.02, H 10.48, N 2.60; found: C 77.76, H 10.61, N 2.44.

\section{$\mathbf{N}^{1}, \quad \mathbf{N}^{7}$-Bis-[(3ß)-3-acetyloxy-olean-12-en-28-oyl]- 1,7-diaminoheptane (8)}

Compound $8(0.12 \mathrm{~g}, 32 \%)$ showed: m.p. $166^{\circ} \mathrm{C}$; $\mathrm{R}_{F}=0.4$ (silica gel, toluene/ethyl acetate/formic $\mathrm{acid} /$ heptane, 80:26:5:1); $[\alpha]_{\mathrm{D}}=+47.82^{\circ}$ (c 0.31, $\left.\mathrm{CHCl}_{3}\right)$;

IR (KBr): $v=3426 b r, 2944 s, 2876 m, 1734 s, 1640 m$, $1524 m, 1466 m, 1370 m, 1248 s, 1212 w, 1148 w$, $1096 w, 1028 m, 1008 m, 986 \mathrm{~m} \mathrm{~cm}^{-1}$;

${ }^{1} \mathrm{H}$ NMR (400 MHz, $\left.\mathrm{CDCl}_{3}\right): \delta=5.90(t, J=5.3 \mathrm{~Hz}$, $2 \mathrm{H}, 2 \times \mathrm{NH}), 5.36(t, J=3.4 \mathrm{~Hz}, 2 \mathrm{H}, 2 \times 12-\mathrm{H}), 4.49$ $(d d, J=9.6,6.3 \mathrm{~Hz}, 2 \mathrm{H}, 2 \times 3-\mathrm{H}), 3.34(d q, J=13.6$, $7.1 \mathrm{~Hz}, 2 \mathrm{H}, 2$ × 33-H), 3.03-2.93 (m, 2H, 2 × 33-H), $2.49(d d, J=12.8,3.5 \mathrm{~Hz}, 2 \mathrm{H}, 2 \times 18-\mathrm{H}), 2.04(s, 6 \mathrm{H}$, $2 \times 32-\mathrm{H}), 1.97(d d, J=13.7,3.6 \mathrm{~Hz}, 2 \mathrm{H}, 2 \times 16-\mathrm{H})$, $1.91(d d, J=8.6,3.3 \mathrm{~Hz}, 2 \mathrm{H}, 2 \times 11-\mathrm{H}), 1.81-1.50$ $(m, 32 \mathrm{H}, 2 \times 1-\mathrm{H}+2 \times 2-\mathrm{H}+2 \times 6-\mathrm{H}+2 \times 7-\mathrm{H}+2 \times$ $9-\mathrm{H}+2 \times 11-\mathrm{H}+2 \times 16-\mathrm{H}+2 \times 19-\mathrm{H}+2 \times 34-\mathrm{H}+2$ x $35-\mathrm{H}+36-\mathrm{H}), 1.45(d d, J=12.8,5.9 \mathrm{~Hz}, 2 \mathrm{H}, 2$ x $15-$ $\mathrm{H}), 1.39(m, 2 \mathrm{H}, 2 \times 22-\mathrm{H}), 1.35(m, 6 \mathrm{H}, 2 \times 6-\mathrm{H}+2$ x 7-H + 2 x 21-H), $1.28(d d, J=12.9,8.0 \mathrm{~Hz}, 2 \mathrm{H}, 2$ x $22-\mathrm{H}), 1.19(d, J=6.3 \mathrm{~Hz}, 4 \mathrm{H}, 2 \times 19-\mathrm{H}+2 \times 21-\mathrm{H})$, $1.15(s, 6 \mathrm{H}, 2 \times 27-\mathrm{H}), 1.04(m, 4 \mathrm{H}, 2 \times 1-\mathrm{H}+2 \times 15-$ $\mathrm{H}), 0.94(s, 6 \mathrm{H}, 2 \times 25-\mathrm{H}), 0.90(s, 6 \mathrm{H} 2 \times 29-\mathrm{H}), 0.90$ $(s, 6 \mathrm{H}, 2 \times 30-\mathrm{H}), 0.87(s, 6 \mathrm{H}, 2 \times 23-\mathrm{H}), 0.85(s, 6 \mathrm{H}$, $2 \times 26-\mathrm{H}), 0.82(s, 2 \mathrm{H}, 2 \times 5-\mathrm{H}), 0.76(s, 6 \mathrm{H}, 2 \times 24-$ H) ppm;

${ }^{13} \mathrm{C}$ NMR (125 MHz, $\left.\mathrm{CDCl}_{3}\right): \delta=178.0$ ( $\left.2 \times \mathrm{C}-28\right)$, 170.9 ( 2 x C-28), 145.1 ( 2 x C-13), 122.5 (2 x C-12), 80.8 ( 2 x C-3), 55.1 ( 2 x C-5), 47.4 (2 x C-9), 46.7 (2 x C-17), 46.2 (2 x C-19), 42.3 (2 x C-18), 42.1 (2 x C-14), 39.3 (2 x C-8), 39.2 ( 2 x C-33), 38.1 ( 2 x C-1), 37.6 ( 2 x C-4), 36.8 ( 2 x C-10), 34.1 ( 2 x C-21), 32.9 (2 x C-29), 32.5 ( 2 x C-7), 32.3 (2 x C-22), 30.7 ( 2 x C-20), 29.3 ( $2 \times$ C-34), 28.9 (C-36), 28.0 (2 x C-23), 27.2 ( 2 x C-27), 27.0 ( 2 x C-15), 25.6 ( 2 x C-35), 23.7 (2 x C-11), 23.5 ( 2 x C-30), 23.5 (2 x C-2), 23.4 ( 2 x C-16), 21.2 (2 x C-32), 18.1 (2 x C-6), $16.9(2 \times$ C-24), 16.6 ( 2 x C-26), 15.4 ( 2 x C-25) ppm;

MS (ESI, MeOH): $m / z=1092.9\left(20 \%,[\mathrm{M}+\mathrm{H}]^{+}\right)$, $1114.8\left(100 \%,[\mathrm{M}+\mathrm{Na}]^{+}\right)$;

analysis calcd for $\mathrm{C}_{71} \mathrm{H}_{114} \mathrm{~N}_{2} \mathrm{O}_{6}$ (1091.68): C 78.11, H 10.53, N 2.57; found: C 77.94, H 10.61, N 2.36.

\section{$\mathbf{N}^{1}, \mathbf{N}^{2}$-Bis-[(3ß)-3-acetyloxy-urs-12-en-28-oyl]-1,2- diaminoethane (9)}

Compound $9(0.28 \mathrm{~g}, 73 \%)$ showed: m.p. $218^{\circ} \mathrm{C}$; $\mathrm{R}_{F}=0.5$ (silica gel, toluene/ethyl acetate/formic $\mathrm{acid} /$ heptane, 80:26:5:1); $[\alpha]_{\mathrm{D}}=+43.5^{\circ}$ (c 0.34, $\mathrm{CHCl}_{3}$ ); 
IR (KBr): $v=3420 b r, 2948 s, 2872 m, 1736 s, 1646 m$, $1522 \mathrm{~m}, 1466 \mathrm{~m}, 1456 \mathrm{~m}, 1390 \mathrm{~m}, 1370 \mathrm{~m}, 1246 \mathrm{~s}$, $1146 w, 1092 w, 1028 m, 1006 w \mathrm{~cm}^{-1}$

${ }^{1} \mathrm{H}$ NMR $\left(400 \mathrm{MHz}, \mathrm{CDCl}_{3}\right): \delta=6.50(m, 2 \mathrm{H}, 2 \mathrm{x}$ $\mathrm{NH}), 5.36(t, J=3.4 \mathrm{~Hz}, 2 \mathrm{H}, 2 \times 12-\mathrm{H}), 4.49(d d, J=$ $9.7,6.1 \mathrm{~Hz}, 2 \mathrm{H}, 2 \times 3-\mathrm{H}), 3.46(d, J=9.0 \mathrm{~Hz}, 2 \mathrm{H}, 2 \mathrm{x}$ $33-\mathrm{H}), 3.08(d, J=8.7 \mathrm{~Hz}, 2 \mathrm{H}, 2 \times 33-\mathrm{H}), 2.04(s, 6 \mathrm{H}$, $2 \times 32-\mathrm{H}), 1.99(d, J=4.1 \mathrm{~Hz}, 2 \mathrm{H}, 2 \times 16-\mathrm{H}), 1.95$ $(d d, J=7.6,3.4 \mathrm{~Hz}, 2 \mathrm{H}, 2 \times 11-\mathrm{H}), 1.90(m, 2 \mathrm{H}, 2 \mathrm{x}$ $18-\mathrm{H}), \quad 1.89-1.82(m, 2 \mathrm{H}, 2$ х 22-H), 1.75-1.60 $(m, 12 \mathrm{H}, 2 \times 1-\mathrm{H}+2 \times 2-\mathrm{H}+2 \times 9-\mathrm{H}+2 \times 11-\mathrm{H}+2 \times$ $16-\mathrm{H}), 1.52(m, 6 \mathrm{H}, 2 \times 15-\mathrm{H}+2 \times 7-\mathrm{H}+2 \times 6-\mathrm{H})$, $1.47-1.40(m, 6 \mathrm{H}, 2 \times 21-\mathrm{H}+2 \times 22-\mathrm{H}+(2 \times 19-\mathrm{H})$, $1.41-1.24(m, 6 \mathrm{H}, 2 \times 6-\mathrm{H}+2 \times 7-\mathrm{H}+2 \times 21-\mathrm{H}), 1.08$ $(s, 6 \mathrm{H}, 2 \times 27-\mathrm{H}), 1.07-1.01(m, 4 \mathrm{H}, 2 \times 1-\mathrm{H}+2 \times$ $15-\mathrm{H}), 0.95(s, 6 \mathrm{H}, 2 \times 30-\mathrm{H}), 0.94(s, 6 \mathrm{H}, 2 \times 25-\mathrm{H})$, $0.88(s, 6 \mathrm{H}, 2 \times 24-\mathrm{H}), 0.86(s, 6 \mathrm{H}, 2 \times 23-\mathrm{H}), 0.85$ $(s, 6 \mathrm{H}, 2 \times 26-\mathrm{H}), 0.82(m, 2 \mathrm{H}, 2 \times 5-\mathrm{H}), 0.75(s, 6 \mathrm{H}$, 2 × 29-H) ppm;

${ }^{13} \mathrm{C} \mathrm{NMR}\left(100 \mathrm{MHz}, \mathrm{CDCl}_{3}\right): \delta=179.3(2 \times \mathrm{C}-28)$, 170.9 ( $2 \times \mathrm{C}-28), 139.3$ ( $2 \times \mathrm{C}-13), 125.8$ ( $2 \times \mathrm{C}-12)$, 80.8 ( $2 \times \mathrm{C}-3), 55.2$ ( $2 \times \mathrm{C}-5), 53.4$ ( $2 \times \mathrm{C}-18), 47.6$ ( $2 \times \mathrm{C}-17), 47.4$ (2 x C-9), 42.3 (2 x C-14), 39.8 (C-33), 39.7 (2 x C-8), 39.5 (2 x C-19), $39.0(2 \times$ C-20), 38.2 ( 2 x C-1), 37.6 ( 2 x C-4), 36.8 ( 2 x C-10), 36.7 ( 2 x C-22), 32.5 ( 2 x C-7), 30.8 ( 2 x C-21), 28.0 ( 2 x C-23), 27.8 ( 2 x C-15), 24.7 (2 x C-16), 23.5 ( $2 \times$ C-27), 23.3 ( $2 \times \mathrm{C}-2), 21.2(2 \times \mathrm{C}-32), 21.1(2 \times$ C-30), 18.1 (2 x C-6), 17.2 (2 x C-24), 16.8 ( 2 x C-29), 16.7 ( 2 x C-26), 15.5 ( 2 x C-25) ppm;

MS (ESI, $\mathrm{MeOH}): m / z=530.5\left(10 \%,[\mathrm{M}+\mathrm{Ca}]^{2+}\right)$, $1021.7\left(100 \%,[\mathrm{M}+\mathrm{H}]^{+}\right), 1043.8\left(80 \%,[\mathrm{M}+\mathrm{Na}]^{+}\right)$; analysis calcd for $\mathrm{C}_{66} \mathrm{H}_{104} \mathrm{~N}_{2} \mathrm{O}_{6}$ (1021.54): C 77.60, H 10.26, N 2.74; found: C 77.45, H 10.41, N 2.51.

\section{$\mathrm{N}^{1}, \mathrm{~N}^{3}$-Bis-[(3ß)-3-acetyloxy-urs-12-en-28-oyl]-1,3- diaminopropane (10)}

Compound $10(0.33 \mathrm{~g}, 88 \%)$ showed: m.p. $206^{\circ} \mathrm{C}$; $\mathrm{R}_{F}=0.5$ (silica gel, toluene/ethyl acetate/formic acid/heptane, 80:26:5:1); $[\alpha]_{\mathrm{D}}=+30.34^{\circ}($ c 0.31 , $\left.\mathrm{CHCl}_{3}\right)$;

IR $(\mathrm{KBr}): v=3393 b r, 2939 m, 2860 m, 1735 s, 1645 m$, $1517 m, 1454 m, 1369 m, 1246 s, 1146 w, 1092 w$,

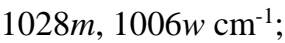

${ }^{1} \mathrm{H}$ NMR $\left(500 \mathrm{MHz}, \mathrm{CDCl}_{3}\right): \delta=6.36(t, J=5.4 \mathrm{~Hz}$, $2 \mathrm{H}, 2 \times \mathrm{NH}), 5.35(t, J=3.5 \mathrm{~Hz}, 2 \mathrm{H}, 2 \times 12-\mathrm{H}), 4.49$ $(d d, J=10.5,5.3 \mathrm{~Hz}, 2 \mathrm{H}, 2 \times 3-\mathrm{H}), 3.24(d q, J=13.1$, $6.6 \mathrm{~Hz}, 2 \mathrm{H}, 2 \mathrm{x} 33-\mathrm{H}), 3.09(d q, J=11.8,5.8 \mathrm{~Hz}, 2 \mathrm{H}$, $2 \times 33-\mathrm{H}), 2.04(s, 6 \mathrm{H}, 2 \times 32-\mathrm{H}), 1.99(d, J=3.9 \mathrm{~Hz}$, $2 \mathrm{H} 2$ x 16-H), 1.94 (ddd, $J=17.8,10.4,2.7 \mathrm{~Hz}, 2 \mathrm{H}, 2$ x 11-H), $1.88(m, 2 \mathrm{H}, 2$ x 18-H), 1.87-1.82 ( $m, 2 \mathrm{H}, 2$ x 22-H), 1.76-1.52 (m, 14H, 2 x 1-H + 2 × $2-\mathrm{H}+2$ x $9-\mathrm{H}+2 \times 11-\mathrm{H}+2 \times 16-\mathrm{H}+2 \times 34-\mathrm{H}), 1.49(\mathrm{~m}, 6 \mathrm{H}, 2$ x $15-\mathrm{H}+2 \times 7-\mathrm{H}+2 \times 6-\mathrm{H}), 1.48-1.40(m, 6 \mathrm{H}, 2 \times$ $21-\mathrm{H}+2 \times 22-\mathrm{H}+(2 \times 19-\mathrm{H}), 1.37-1.26(m, 6 \mathrm{H}, 2 \times$ $6-\mathrm{H}+2 \times 7-\mathrm{H}+2 \times 21-\mathrm{H}), 1.05(s, 6 \mathrm{H}, 2 \times 27-\mathrm{H})$, $1.07-1.01(m, 4 \mathrm{H}, 2 \times 1-\mathrm{H}+2 \times 15-\mathrm{H}), 0.95(s, 6 \mathrm{H}, 2$ х 30-H), $0.93(s, 6 \mathrm{H}, 2 \times 25-\mathrm{H}), 0.88(s, 6 \mathrm{H}, 2 \times$ $24-\mathrm{H}), 0.87(s, 6 \mathrm{H}, 2 \times 23-\mathrm{H}), 0.86(s, 6 \mathrm{H}, 2 \times 26-\mathrm{H})$, $0.82(s, 2 \mathrm{H}, 2 \times 5-\mathrm{H}), 0.76(s, 6 \mathrm{H} 2 \times 29-\mathrm{H}) \mathrm{ppm}$;
${ }^{13} \mathrm{C}$ NMR (125 MHz, $\left.\mathrm{CDCl}_{3}\right): \delta=178.5(2 \times \mathrm{C}-28)$, 170.9 ( 2 x C-28), 139.4 ( 2 x C-13), 125.6 ( 2 x C-12), 80.8 ( 2 x C-3), 55.2, (2 x C-5), 53.5 (2 x C-18), 47.7 (2 x C-17), 47.4 (2 x C-9), 42.3 (2 x C-14), 39.7 ( 2 x C-8), 39.5 (2 x C-19), 38.9 (2 x C-20), 38.3 ( 2 x C-1), 37.6 ( 2 x C-4), 37.4 ( 2 x C-10), 36.8 ( 2 x C-22), 36.3 (C-33), 32.7 (2 x C-7), 30.9 ( 2 x C-21), 29.6 (C-34), 28.0 ( 2 x C-23), 27.8 ( 2 x C-15), 24.8 ( 2 x C-16), 23.4 (2 x C-2), 23.2 (2 x C-27), 21.2 (2 x C-32), 21.1 ( 2 x C-30), 18.1 (2 x C-6), 17.2 (2 x C-24), 17.0 ( 2 x $\mathrm{C}-29), 16.8$ ( $2 \times \mathrm{C}-26), 15.5$ ( $2 \times \mathrm{C}-25) \mathrm{ppm}$;

MS (ESI, MeOH): $m / z=1035.7\left(100 \%,[\mathrm{M}+\mathrm{H}]^{+}\right)$, $1057.8\left(70 \%,[\mathrm{M}+\mathrm{Na}]^{+}\right)$;

analysis calcd for $\mathrm{C}_{67} \mathrm{H}_{106} \mathrm{~N}_{2} \mathrm{O}_{6}$ (1035.57): C 77.71, H 10.32, N 2.71; found: C 77.58, H 10.46, N 2.53.

\section{$\mathrm{N}^{1}, \mathrm{~N}^{4}$-Bis-[(3ß)-3-acetyloxy-urs-12-en-28-oyl]-1,4- diaminobutane (11)}

Compound $11(0.33 \mathrm{~g}, 87 \%)$ showed: m.p. $206^{\circ} \mathrm{C}$; $\mathrm{R}_{F}=0.4$ (silica gel, toluene/ethyl acetate/formic $\mathrm{acid} /$ heptane, 80:26:5:1); $[\alpha]_{\mathrm{D}}=+30.2^{\circ}$ (c 0.32 , $\left.\mathrm{CHCl}_{3}\right)$;

IR (KBr): $v=3445 b r, 2942 m, 2859 m, 1735 s, 1637 m$, $1421 m, 1325 m, 1245 s, 1147 w, 1045 w, 1028 m, 1005 w$ $\mathrm{cm}^{-1}$;

${ }^{1} \mathrm{H}$ NMR $\left(500 \mathrm{MHz}, \mathrm{CDCl}_{3}\right): \delta=5.99(t, J=5.1 \mathrm{~Hz}$, $2 \mathrm{H}, 2 \times \mathrm{NH}), 5.31(t, J=3.9 \mathrm{~Hz}, 2 \mathrm{H}, 2 \times 12-\mathrm{H}), 4.49$ $(d d, J=10.5,5.4 \mathrm{~Hz}, 2 \mathrm{H}, 2 \times 3-\mathrm{H}), 3.36(d d, J=13.4$, $6.5 \mathrm{~Hz}, 2 \mathrm{H}, 2 \times 33-\mathrm{H}), 2.99(d q, J=11.0,6.1 \mathrm{~Hz}, 2 \mathrm{H}$, $2 \times 33-\mathrm{H}), 2.05(s, 6 \mathrm{H}, 2 \times 32-\mathrm{H}), 2.00-1.98(m, 2 \mathrm{H}$, $2 \times 16-\mathrm{H}), 1.95(d d d, J=13.5,7.0,3.5 \mathrm{~Hz}, 2 \mathrm{H}, 2 \times$ 11-H), 1.90-1.87 ( $m, 2 \mathrm{H}, 2$ x 18-H), $1.86(m, 2 \mathrm{H}, 2$ x $22-\mathrm{H}), 1.76-1.55(m, 16 \mathrm{H}, 2 \times 1-\mathrm{H}+2 \times 2-\mathrm{H}+2 \times$ $9-\mathrm{H}+2 \times 11-\mathrm{H}+2 \times 16-\mathrm{H}+2 \times 34-\mathrm{H}), 1.54-1.49$ $(m, 6 \mathrm{H}, 2 \times 15-\mathrm{H}+2 \times 7-\mathrm{H}+2 \times 6-\mathrm{H}), 1.45(m, 6 \mathrm{H}$, $2 \times 21-\mathrm{H}+2 \times 22-\mathrm{H}+(2 \times 19-\mathrm{H}), 1.41-1.26(m, 6 \mathrm{H}$, $2 \times 6-\mathrm{H}+2 \times 7-\mathrm{H}+2 \times 21-\mathrm{H}), 1.09(s, 6 \mathrm{H}, 2 \times 27-\mathrm{H})$, $1.07-1.02(m, 4 \mathrm{H}, 2 \times 1-\mathrm{H}+2 \times 15-\mathrm{H}), 0.95(s, 6 \mathrm{H}, 2$ x 30-H), $0.88(s, 6 \mathrm{H}, 2$ x 24-H), $0.86(s, 6 \mathrm{H}, 2$ × $23-$ $\mathrm{H}), 0.85(s, 6 \mathrm{H}, 2 \times 26-\mathrm{H}), 0.76(s, 6 \mathrm{H} 2 \times 29-\mathrm{H}) \mathrm{ppm}$; ${ }^{13} \mathrm{C}$ NMR $\left(125 \mathrm{MHz}, \mathrm{CDCl}_{3}\right): \delta=178.0(2 \times \mathrm{C}-28)$, 170.9 ( 2 x C-28), 140.0 ( $2 \times$ C-13), 125.4 ( $2 \times$ C-12), 80.8 ( 2 x C-3), 55.2 (2 x C-5), 53.8 (2 x C-18), 47.7 (2 x C-17), 47.4 (2 x C-9), 42.4 (2 x C-14), 39.7 ( 2 x C-33), 39.7 ( $2 \times \mathrm{C}-8), 39.5$ ( $2 \times \mathrm{C}-19), 39.0$ ( $2 \times$ C-20), 38.9 ( 2 x C-1), 37.6 ( 2 x C-4), 36.8 ( 2 x C-10), 36.7 ( 2 x C-22), 32.6 ( 2 x C-7), 30.8 ( 2 x C-21), 28.0 (2 x C-23), 27.8 (2 x C-15), 27.0 (2 x C-34), ( 2 x C-16), 23.5 ( 2 x C-27), 23.1 ( 2 x C-2), 21.2 ( 2 x C32), 21.1 ( 2 x C-30), 17.7 ( 2 × C-6), 17.2 ( $2 \times$ C-24), 16.9 ( 2 x C-29), 16.7 ( 2 x C-26), 15.5 ( 2 x C-25) ppm; MS (ESI, MeOH): $m / z=1049.7\left(100 \%,[\mathrm{M}+\mathrm{H}]^{+}\right)$, $545.0\left(20 \%,[\mathrm{M}+\mathrm{Ca}]^{2+}\right), 1069.3\left(54 \%,[2 \mathrm{M}+\mathrm{Ca}]^{2+}\right)$, $1071.8\left(40 \%,[\mathrm{M}+\mathrm{Na}]^{+}\right)$;

analysis calcd for $\mathrm{C}_{68} \mathrm{H}_{108} \mathrm{~N}_{2} \mathrm{O}_{6}$ (1049.60): C 77.81, H 10.37, N 2.67; found: C 77.69, H 10.57, N 2.41.

\section{$\mathrm{N}^{1}, \mathrm{~N}^{5}$-Bis-[(3ß)-3-acetyloxy-urs-12-en-28-oyl]-1,5-} diaminopentane (12)

Compound $12(0.31 \mathrm{~g}, 81 \%)$ showed: m.p. $188^{\circ} \mathrm{C}$; $\mathrm{R}_{F}=0.4$ (silica gel, toluene/ethyl acetate/formic 
$\mathrm{acid} /$ heptane, 80:26:5:1); $[\alpha]_{\mathrm{D}}=+36.32^{\circ}$ (c 0.31 , $\left.\mathrm{CHCl}_{3}\right)$;

IR (KBr): $v=3403 b r, 2926 m, 2871 m, 1734 s, 1638 m$, $1514 m, 1454 m, 1369 m, 1243 s, 1147 w, 1093 w$,

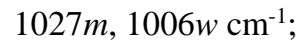

${ }^{1} \mathrm{H}$ NMR $\left(500 \mathrm{MHz}, \mathrm{CDCl}_{3}\right): \delta=5.99(t, J=5.1 \mathrm{~Hz}$, $2 \mathrm{H}, 2 \times \mathrm{NH}), 5.31(t, J=3.9 \mathrm{~Hz}, 2 \mathrm{H}, 2 \times 12-\mathrm{H}), 4.49$ $(d d, J=10.5,5.4 \mathrm{~Hz}, 2 \mathrm{H}, 2 \times 3-\mathrm{H}), 3.36(d d, J=13.4$, $6.5 \mathrm{~Hz}, 2 \mathrm{H}, 2 \times 33-\mathrm{H}), 2.99(d q, J=11.0,6.1 \mathrm{~Hz}, 2 \mathrm{H}$, $2 \times 33-\mathrm{H}), 2.05(s, 6 \mathrm{H}, 2 \times 32-\mathrm{H}), 2.00-1.98(m, 2 \mathrm{H}$, $2 \times 16-\mathrm{H}), 1.95(d d d, J=13.5,7.0,3.5 \mathrm{~Hz}, 2 \mathrm{H}, 2 \times$ $11-\mathrm{H}), 1.90-1.87(m, 2 \mathrm{H}, 2 \times 18-\mathrm{H}), 1.86(m, 2 \mathrm{H}, 2 \mathrm{x}$ $22-\mathrm{H}), 1.76-1.55(m, 18 \mathrm{H}, 2 \times 1-\mathrm{H}+2 \times 2-\mathrm{H}+2 \times$ $9-\mathrm{H}+2 \times 11-\mathrm{H}+2 \times 16-\mathrm{H}+2 \times 34-\mathrm{H}+35-\mathrm{H})$, $1.54-1.49(m, 6 \mathrm{H}, 2 \times 15-\mathrm{H}+2 \times 7-\mathrm{H}+2 \times 6-\mathrm{H}), 1.45$ $(m, 6 \mathrm{H}, 2 \times 21-\mathrm{H}+2 \times 22-\mathrm{H}+2 \times 19-\mathrm{H}), 1.41-1.26$ $(m, 6 \mathrm{H}, 2 \times 6-\mathrm{H}+2 \times 7-\mathrm{H}+2 \times 21-\mathrm{H}), 1.09(s, 6 \mathrm{H}$, $2 \times 27-\mathrm{H}), 1.07-1.02(m, 4 \mathrm{H}, 2 \times 1-\mathrm{H}+2 \times 15-\mathrm{H})$, $0.95(s, 6 \mathrm{H}, 2 \times 30-\mathrm{H}), 0.88(s, 6 \mathrm{H}, 2 \times 24-\mathrm{H}), 0.86$ $(s, 6 \mathrm{H}, 2 \times 23-\mathrm{H}), 0.85(s, 6 \mathrm{H}, 2 \times 26-\mathrm{H}), 0.76(s, 6 \mathrm{H}$ $2 \times 29-\mathrm{H}) \mathrm{ppm}$;

${ }^{13} \mathrm{C} \mathrm{NMR}\left(125 \mathrm{MHz}, \mathrm{CDCl}_{3}\right): \delta=178.2$ ( $\left.2 \times \mathrm{C}-28\right)$, 170.9 ( 2 x C-28), 140.0 ( 2 x C-13), 125.4 ( 2 x C-12), 80.7 ( $2 \times \mathrm{C}-3), 55.2$ (2 x C-5), 53.9 (2 x C-18), 47.6 (2 x C-17), 47.4 (2 x C-9), 42.5 (2 x C-14), 39.7 (2 x C-8), 39.5 (2 x C-19), 39.3 (2 x C-33), 39.1 (2 x C-20), 38.3 ( 2 x C-1), 37.6 ( 2 x C-4), 37.2 ( 2 x C-10), 36.8 ( $2 \times \mathrm{C}-22), 32.6$ ( $2 \times \mathrm{C}-7), 30.8$ ( $2 \times \mathrm{C}-21), 29.0$ (2 x C-34), 28.0 (2 x C-23), 27.8 ( 2 x C-15), 24.8 ( 2 x C-16), 24.5 (C-35), 23.4 (2 x C-2), 23.1 ( 2 x C-27), 21.2 ( 2 x C-32), 21.1 ( 2 x C-30), 18.1 ( 2 x C-6), 17.2 (2 x C-24), 16.9 (2 x C-29), 16.7 (2 x C-26), 15.5 ( 2 x C-25) ppm;

MS (ESI, MeOH): $m / z=1063.7\left(100 \%,[\mathrm{M}+\mathrm{H}]^{+}\right)$, $1085.8\left(90 \%,[\mathrm{M}+\mathrm{Na}]^{+}\right), 551.5\left(10 \%,[\mathrm{M}+\mathrm{Ca}]^{2+}\right)$; analysis calcd for $\mathrm{C}_{69} \mathrm{H}_{110} \mathrm{~N}_{2} \mathrm{O}_{6}(1063.62)$ : C 77.92, $\mathrm{H}$ 10.42, N 2.63; found: C 77.63, H 10.69, N 2.50.

\section{$N^{1}, N^{6}$-Bis-[(3及)-3-acetyloxy-urs-12-en-28-oyl]-1,6- diaminohexane (13)}

Compound $13(0.33 g, 85 \%)$ showed: m.p. $162^{\circ} \mathrm{C}$; $\mathrm{R}_{F}=0.6$ (silica gel, toluene/ethyl acetate/formic $\mathrm{acid} /$ heptane, 80:26:5:1); $[\alpha]_{\mathrm{D}}=+38.90^{\circ}$ (c 0.32 , $\left.\mathrm{CHCl}_{3}\right)$;

IR (KBr): $v=3428 b r, 2946 m, 2872 m, 1736 s, 1646 m$, $1522 m, 1456 m, 1370 m, 1246 s, 1148 w, 1092 w$,

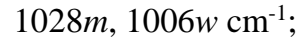

${ }^{1} \mathrm{H}$ NMR $\left(500 \mathrm{MHz}, \mathrm{CDCl}_{3}\right): \delta=5.94(t, J=5.5 \mathrm{~Hz}$, $2 \mathrm{H}, 2 \times \mathrm{NH}), 5.31-5.29(m, 2 \mathrm{H}, 2 \times 12-\mathrm{H}), 4.49$ (dd, $J=10.5,5.3 \mathrm{~Hz}, 2 \mathrm{H}, 2 \times 3-\mathrm{H}), 3.30(d q, J=13.6,6.9$ $\mathrm{Hz}, 2 \mathrm{H}, 2$ x 33-H), 3.03-2.95 (m, 2H, 2 × 33-H), 2.05 $(s, 6 \mathrm{H}, 2 \times 32-\mathrm{H}), 2.02-1.98(m, 2 \mathrm{H}, 2 \times 16-\mathrm{H}), 1.95$ $(d d d, J=14.9,6.2,3.3 \mathrm{~Hz}, 2 \mathrm{H}, 2 \times 11-\mathrm{H}), 1.90-1.87$ ( $m, 4 \mathrm{H}, 2 \times 18-\mathrm{H}+2 \times 22-\mathrm{H}), 1.77-1.55(m, 20 \mathrm{H}, 2 \times$ $1-\mathrm{H}+2 \times 2-\mathrm{H}+2 \times 9-\mathrm{H}+2 \times 11-\mathrm{H}+2 \times 16-\mathrm{H}+2 \times$ $34-\mathrm{H}+(2 \times 35-\mathrm{H}), 1.50(\mathrm{~m}, 6 \mathrm{H}, 2 \times 15-\mathrm{H}+2 \times 7-\mathrm{H}+$ $2 \times 6-\mathrm{H}), 1.48-1.39(m, 6 \mathrm{H}, 2 \times 21-\mathrm{H}+2 \times 22-\mathrm{H}+$ $2 \times 19-\mathrm{H}), 1.39-1.27(m, 6 \mathrm{H}, 2 \times 6-\mathrm{H}+2 \times 7-\mathrm{H}+2 \times$ $21-\mathrm{H}), 1.09(s, 6 \mathrm{H}, 2 \times 27-\mathrm{H}), 1.07-1.01(\mathrm{~m}, 4 \mathrm{H}, 2 \mathrm{x}$ $1-\mathrm{H}+2 \times 15-\mathrm{H}), 0.95(s, 6 \mathrm{H}, 2 \times 30-\mathrm{H}), 0.88(s, 6 \mathrm{H}$,
$2 \times 24-\mathrm{H}), 0.87(s, 6 \mathrm{H}, 2 \times 23-\mathrm{H}), 0.86(s, 6 \mathrm{H}, 2 \times 26-$ $\mathrm{H}), 0.82(m, 2 \mathrm{H}, 2 \times 5-\mathrm{H}), 0.77(s, 6 \mathrm{H} 2 \times 29-\mathrm{H}) \mathrm{ppm}$; ${ }^{13} \mathrm{C}$ NMR (125 MHz, $\left.\mathrm{CDCl}_{3}\right): \delta=178.2(2 \times \mathrm{C}-28)$, 170.9 ( 2 x C-28), 140.0 ( 2 x C-13), 125.4 ( 2 x C-12), 80.8 ( 2 x C-3), 55.2 ( 2 x C-5), 53.9 ( 2 x C-18), 47.7 (2 x C-17), 47.4 (2 x C-9), 42.5 (2 x C-14), 39.7 ( 2 x C-8), 39.5 (2 x C-19), 39.2 (2 x C-33), 39.1 ( 2 x C-20), 38.3 ( 2 x C-1), 37.6 ( 2 x C-4), 37.2 ( 2 x C-10), 36.7 ( 2 x C-22), 32.6 ( 2 x C-7), 30.8 ( 2 x C-21), 29.2 (2 x C-34), 28.0 (2 x C-23), 27.8 ( 2 x C-15), 24.8 ( 2 x C-16), $24.5(2 \times \mathrm{C}-35), 23.4(2 \times \mathrm{C}-2), 23.2(2 \times$ C-27), 21.2 ( $2 \times \mathrm{C}-32), 21.1$ ( $2 \times \mathrm{C}-30), 18.1(2 \times$ C-6), 17.2 (2 x C-24), 16.9 ( 2 x C-29), 16.7 (2 x C26), 15.5 ( $2 \times \mathrm{C}-25) \mathrm{ppm}$;

MS (ESI, MeOH): $m / z=1077.7\left(100 \%,[\mathrm{M}+\mathrm{H}]^{+}\right)$, $1099.8\left(72 \%,[\mathrm{M}+\mathrm{Na}]^{+}\right), 558.5\left(5 \%,[\mathrm{M}+\mathrm{Ca}]^{2+}\right)$; analysis calcd for $\mathrm{C}_{70} \mathrm{H}_{112} \mathrm{~N}_{2} \mathrm{O}_{6}$ (1077.65): C 78.02, H 10.48, N 2.60; found: C 77.88, H 10.69, N 2.41.

\section{$\mathbf{N}^{1}, \mathbf{N}^{7}$-Bis-[(3ß)-3-acetyloxy-urs-12-en-28-oyl]-1,7- diaminoheptane (14)}

Compound $14(0.23 \mathrm{~g}, 56 \%)$ showed: m.p. $176^{\circ} \mathrm{C}$; $\mathrm{R}_{F}=0.4$ (silica gel, toluene/ethyl acetate/formic $\mathrm{acid} /$ heptane, 80:26:5:1); $[\alpha]_{\mathrm{D}}=+36.18^{\circ}($ c 0.33 , $\left.\mathrm{CHCl}_{3}\right)$;

IR (KBr): $v=3418 b r, 2925 m, 2855 m, 1735 s, 1655 m$, $1516 m, 1454 m, 1369 m, 1243 s, 1147 w, 1092 w$, $1027 \mathrm{~m}, 1005 \mathrm{w} \mathrm{cm} \mathrm{cm}^{-1}$;

${ }^{1} \mathrm{H}$ NMR (400 MHz, $\left.\mathrm{CDCl}_{3}\right): \delta=5.91(t, J=5.4 \mathrm{~Hz}$, $2 \mathrm{H}, 2 \times \mathrm{NH}), 5.30(m, 2 \mathrm{H}, 2 \times 12-\mathrm{H}), 4.52-4.47$ $(m, 2 \mathrm{H}, 2 \times 3-\mathrm{H}), 3.31(d q, J=13.5,7.0 \mathrm{~Hz}, 2 \mathrm{H}, 2 \times$ 33-H), 3.04-2.95 (m, 2H, 2 х 33-H), 2.04 ( $s, 6 \mathrm{H}, 2$ х $32-\mathrm{H}), 2.00(d, J=4.2 \mathrm{~Hz}, 2 \mathrm{H}, 2 \times 16-\mathrm{H}), 1.99-1.91$ $(m, 2 \mathrm{H}, 2 \times 11-\mathrm{H}), 1.89-1.80(m, 4 \mathrm{H}, 2 \times 18-\mathrm{H}+2 \times$ $22-\mathrm{H}), 1.77-1.53(m, 22 \mathrm{H}, 2 \times 1-\mathrm{H}+2 \times 2-\mathrm{H}+2 \times$ $9-\mathrm{H}+2 \times 11-\mathrm{H}+2 \times 16-\mathrm{H}+2 \times 34-\mathrm{H}+2 \times 35-\mathrm{H}+$ $36-\mathrm{H}), 1.50(m, 6 \mathrm{H}, 2 \times 15-\mathrm{H}+2 \times 7-\mathrm{H}+2 \times 6-\mathrm{H})$, $1.49-1.41(m, 6 \mathrm{H}, 2 \times 21-\mathrm{H}+2 \times 22-\mathrm{H}+2 \times 19-\mathrm{H})$, $1.40-1.25(m, 6 \mathrm{H}, 2 \times 6-\mathrm{H}+2 \times 7-\mathrm{H}+2 \times 21-\mathrm{H}), 1.09$ $(s, 6 \mathrm{H}, 2 \times 27-\mathrm{H}), 1.05(m, 4 \mathrm{H}, 2 \times 1-\mathrm{H}+2 \times 15-\mathrm{H})$, $0.95(s, 6 \mathrm{H}, 2 \times 30-\mathrm{H}), 0.88(s, 6 \mathrm{H}, 2 \times 24-\mathrm{H}), 0.87$ $(s, 6 \mathrm{H}, 2 \times 23-\mathrm{H}), 0.86(s, 6 \mathrm{H}, 2 \times 26-\mathrm{H}), 0.81(m, 2 \mathrm{H}$, $2 \times 5-\mathrm{H}), 0.78(s, 6 \mathrm{H} 2 \times 29-\mathrm{H}) \mathrm{ppm}$;

${ }^{13} \mathrm{C}$ NMR (100 MHz, $\left.\mathrm{CDCl}_{3}\right): \delta=178.1(2 \times \mathrm{C}-28)$, 170.9 ( 2 x C-28), 140.1 ( 2 × C-13), 125.4 ( $2 \times$ C-12), 80.8 ( 2 x C-3), 55.2 ( 2 x C-5), 54.0 ( $2 \times$ C-18), 47.6 (2 x C-17), 47.4 (2 x C-9), 42.5 (2 x C-14), 39.7 ( 2 x C-8), 39.5 (2 x C-19), 39.4 ( 2 x C-33), 39.1 ( 2 x C-20), 38.3 ( 2 x C-1), 37.6 ( 2 x C-4), 37.1 ( 2 x C-10), 36.8 ( 2 x C-22), 32.6 ( 2 x C-7), 30.8 ( 2 × C-21), 29.2 (2 x C-34), 28.9 (C-36), 28.0 (2 x C-23), 27.8 ( 2 x C-15), 27.0 ( $2 \times$ C-35), 24.8 ( 2 x C-16), 23.4 ( 2 x C2), 23.1 ( $2 \times \mathrm{C}-27), 21.2$ ( $2 \times \mathrm{C}-32) 21.1(2 \times \mathrm{C}-30)$, 18.1(2 x C-6), 17.2 (2 x C-24), 16.9 (2 x C-29), 16.7 (2 x C-26), 15.5 (2 x C-25) ppm;

MS (ESI, MeOH): $m / z=1091.7\left(100 \%,[\mathrm{M}+\mathrm{H}]^{+}\right)$, $1113.0\left(92 \%,[\mathrm{M}+\mathrm{Na}]^{+}\right), 565.9\left(5 \%,[\mathrm{M}+\mathrm{Ca}]^{2+}\right)$; analysis calcd for $\mathrm{C}_{71} \mathrm{H}_{114} \mathrm{~N}_{2} \mathrm{O}_{6}$ (1091.68): C 78.11, H 10.53, N 2.57; found: C 78.01, H 10.64, N 2.38. 


\section{$\mathrm{N}^{1}, \mathrm{~N}^{2}$-Bis-[(3ß)-3-hydroxy-olean-12-en-28-oyl]- 1,2-diaminoethane (15)}

Compound 15 (0.22 g, 59\%) showed: m.p. $239^{\circ} \mathrm{C}$; $\mathrm{R}_{F}=0.5$ (silica gel, toluene/ethyl acetate/formic acid/heptane, 80:26:5:1); $[\alpha]_{\mathrm{D}}=+51.41^{\circ}($ c 0.35 , $\left.\mathrm{CHCl}_{3}\right)$;

IR (KBr): $v=3393 m, 2927 m, 2865 w, 1640 m, 1518 m$, $1463 m, 1386 w, 1364 w, 1262 w, 1094 w, 1029 m, 996 m$ $\mathrm{cm}^{-1} ;{ }^{1} \mathrm{H}$ NMR $\left(400 \mathrm{MHz}, \mathrm{CDCl}_{3}\right): \delta=6.55(s, 2 \mathrm{H}, 2$ $\mathrm{x} \mathrm{NH}), 5.40(t, J=3.3 \mathrm{~Hz}, 2 \mathrm{H}, 2 \mathrm{x} 12-\mathrm{H}), 3.46(d, J=$ $9.3 \mathrm{~Hz}, 2 \mathrm{H},(2 \times 31-\mathrm{H}), 3.21(d d, J=11.1,4.6 \mathrm{~Hz}, 2 \mathrm{H}$, $2 \times 3-\mathrm{H}), 3.12(d, J=8.7 \mathrm{~Hz}, 2 \mathrm{H},(2 \times 31-\mathrm{H}), 2.54(d d$, $J=13.1,3.4 \mathrm{~Hz}, 2 \mathrm{H}, 2 \times 18-\mathrm{H}), 2.00(d d, J=13.7,3.9$ $\mathrm{Hz}, 2 \mathrm{H}, 2$ x 16-H), $1.93(d d, J=11.0,7.3 \mathrm{~Hz}, 2 \mathrm{H}, 2 \mathrm{x}$ $11-\mathrm{H}), 1.79-1.53(\mathrm{~m}, 18 \mathrm{H}, 2 \times 1-\mathrm{H}+2 \times 2-\mathrm{H}+2 \times$ $6-\mathrm{H}+2 \times 7-\mathrm{H}+2 \times 9-\mathrm{H}+2 \times 11-\mathrm{H}+2 \times 16-\mathrm{H}+2 \times$ $19-\mathrm{H}), \quad 1.52-1.50(m, 2 \mathrm{H}, 2 \times 15-\mathrm{H}), 1.49-1.39$ $(m, 2 \mathrm{H}, 2 \times 22-\mathrm{H}), 1.39-1.29(m, 6 \mathrm{H}, 2 \times 6-\mathrm{H}+2 \times$ $7-\mathrm{H}+2 \times 21-\mathrm{H}), 1.29-1.22(m, 2 \mathrm{H}, 2 \times 22-\mathrm{H}), 1.21-$ $1.17(m, 4 \mathrm{H}, 2 \times 19-\mathrm{H}+2 \times 21-\mathrm{H}), 1.15(s, 6 \mathrm{H}, 2 \times$ $27-\mathrm{H}), 1.02(d d, J=14.2,3.7 \mathrm{~Hz}, 4 \mathrm{H}, 2 \times 1-\mathrm{H}+2 \mathrm{x}$ $15-\mathrm{H}), 0.98$ ( $s, 6 \mathrm{H}, 2 \times 23-\mathrm{H}), 0.90(s, 6 \mathrm{H}, 2 \times 25-\mathrm{H})$, $0.89(s, 6 \mathrm{H},(2 \times 30-\mathrm{H}), 0.89(s, 6 \mathrm{H}, 2 \times 29-\mathrm{H}), 0.77$ $(s, 6 \mathrm{H}, 2 \times 24-\mathrm{H}), 0.73(s, 6 \mathrm{H}, 2 \times 26-\mathrm{H}), 0.71(s, 2 \mathrm{H}$, 2 x 5-H ppm;

${ }^{13} \mathrm{C} \mathrm{NMR}\left(100 \mathrm{MHz}, \mathrm{CDCl}_{3}\right): \delta=179.3(2 \times \mathrm{C}-28)$, 144.4 ( 2 x C-13), 123.1 ( 2 x C-12), 78.9 ( $2 \times$ C-3), 55.1 ( 2 x C-5), 47.5 ( 2 x C-9), 46.6 ( 2 x C-17), 46.1 (2 x C-19), 41.9 (2 x C-18), 41.9 (2 x C-14), 40.1 ( 2 x C-31), 39.3 ( 2 x C-8), 38.7 ( $2 \times$ C-1), 38.4 ( $2 \times$ C-4), 36.9 ( 2 x C-10), 34.1 ( 2 x C-21), 32.9 ( 2 x C-29), 32.7 ( 2 x C-7), 32.3 ( $2 \times$ C-22), 30.6 ( $2 \times$ C-20), $28.0(2 \times$ C-23), 27.3 ( $2 \times \mathrm{C}-15), 27.1(2 \times \mathrm{C}-2), 25.8(2 \times$ C-27), 23.6 ( 2 x C-11), 23.5 ( 2 x C-30), 23.5 ( 2 x C-16), 18.2 (2 x C-6), 16.8 ( 2 x C-26), 15.5 ( 2 x C24), 15.3 (2 x C-25) ppm;

MS (ESI, MeOH): $m / z=938.1\left(42 \%,[\mathrm{M}+\mathrm{H}]^{+}\right), 960.2$ $\left(100 \%,[\mathrm{M}+\mathrm{Na}]^{+}\right)$;

analysis calcd for C62H100N2O4 (937.47): C 79.43, H 10.75, N 2.99; found: C 79.17, H 10.91, N 2.76.

\section{$\mathrm{N}^{1}, \mathrm{~N}^{3}$-Bis-[(3ß)-3-hydroxy-olean-12-en-28-oyl]- 1,3-diaminopropane (16)}

Compound $16(0.22 \mathrm{~g}, 59 \%)$ showed: m.p. $218^{\circ} \mathrm{C}$; $\mathrm{R}_{F}=0.3$ (silica gel, toluene/ethyl acetate/formic acid/heptane, 80:26:5:1); $[\alpha]_{\mathrm{D}}=+50.78^{\circ}$ (c 0.30 , $\left.\mathrm{CHCl}_{3}\right)$;

IR $(\mathrm{KBr}): v=3374 b r, 2928 m, 2864 w, 1632 s, 1527 s$, $1465 m, 1432 m, 1380 w, 1363 w, 1262 w, 1094 w$, $1045 m, 1030 m, 998 w, 773 w \quad \mathrm{~cm}^{-1}$;

${ }^{1} \mathrm{H}$ NMR $\left(500 \mathrm{MHz}, \mathrm{CDCl}_{3}\right): \delta=6.47(t, J=5.1 \mathrm{~Hz}$, $2 \mathrm{H}, 2 \times \mathrm{NH}), 5.41(t, J=3.5 \mathrm{~Hz}, 2 \mathrm{H}, 2 \times 12-\mathrm{H}), 3.27$ $(d q, J=13.4,6.8 \mathrm{~Hz}, 2 \mathrm{H}, 2 \times 31-\mathrm{H}), 3.21(d d, J=$ $11.4,4.3 \mathrm{~Hz}, 2 \mathrm{H}, 2$ x 3-H), $3.13(d q, J=12.0,5.9 \mathrm{~Hz}$, $2 \mathrm{H}, 2 \times 31-\mathrm{H}), 2.65(\mathrm{dd}, J=13.0,4.0 \mathrm{~Hz}, 2 \mathrm{H}, 2 \times$ $18-\mathrm{H}), 2.02-1.93(m, 2 \mathrm{H}, 2 \times 16-\mathrm{H}), 1.93-1.87$ $(m, 2 \mathrm{H}, 2 \times 11-\mathrm{H}), 1.79-1.50(m, 20 \mathrm{H}, 2 \times 1-\mathrm{H}+2 \times$ $2-\mathrm{H}+2 \times 6-\mathrm{H}+2 \times 7-\mathrm{H}+2 \times 9-\mathrm{H}+2 \times 11-\mathrm{H}+2 \times$ $15-\mathrm{H}+2 \times 16-\mathrm{H}+2 \times 19-\mathrm{H}+32-\mathrm{H}), 1.44(d d, J=$ $12.2,3.1 \mathrm{~Hz}, 2 \mathrm{H}, 2 \times 22-\mathrm{H}), 1.41-1.30(m, 6 \mathrm{H}, 2 \times$ $6-\mathrm{H}+2 \times 7-\mathrm{H}+2 \times 21-\mathrm{H}), 1.30-1.24(\mathrm{~m}, 2 \mathrm{H}, 2$ × $22-$
H), 1.22-1.17 ( $m, 4 \mathrm{H}, 2 \times 19-\mathrm{H}+2 \times 21-\mathrm{H}), 1.16$ $(s, 6 \mathrm{H}, 2 \times 27-\mathrm{H}), 1.04(d t, J=13.5,2.9 \mathrm{~Hz}, 4 \mathrm{H}, 2 \times$ $1-\mathrm{H}+2 \times 15-\mathrm{H}), 0.99(s, 6 \mathrm{H}, 2 \times 23-\mathrm{H}), 0.92(s, 6 \mathrm{H}$, $2 \times 25-\mathrm{H}), 0.90(s, 6 \mathrm{H},(2 \times 30-\mathrm{H}), 0.90(s, 6 \mathrm{H}, 2 \times$ $29-\mathrm{H}), 0.78$ ( $s, 6 \mathrm{H}, 2 \times 24-\mathrm{H}), 0.74(s, 6 \mathrm{H}, 2 \times 26-\mathrm{H})$, $0.72(s, 2 \mathrm{H}, 2 \times 5-\mathrm{H}) \mathrm{ppm}$;

${ }^{13} \mathrm{C} \mathrm{NMR}\left(125 \mathrm{MHz}, \mathrm{CDCl}_{3}\right): \delta=178.5(2 \times \mathrm{C}-28)$, 144.5 ( 2 x C-13), 122.8 ( 2 × C-12), 78.9 ( $2 \times$ C-3), 55.1 ( 2 x C-5), 47.5 ( 2 x C-9), 46.7 (2 x C-17), 46.3 ( $2 \times \mathrm{C}-19), 41.9$ (2 x C-18), 41.84 (2 x C-14), 39.3 ( 2 x C-8), 38.7 ( $2 \times \mathrm{C}-1), 38.4$ (2 x C-4), $36.9(2 \times$ C-10), 36.1 ( 2 x C-31), 34.2 ( $2 \times$ C-21), 33.01 ( $2 \times$ C-29), 32.9 ( $2 \times$ C-7), 32.4 ( 2 x C-22), 30.7 ( 2 x C-20), 29.6 (CH2, C32), 28.0 (2 x C-23), 27.3 (2 x C-15), 27.15 (2 x C-2), 25.7 ( $2 \times \mathrm{C}-27), 23.6(2 \times$ C-11), 23.6 ( $2 \times$ C-30), 23.5 ( $2 \times$ C-16), $18.2(2 \times$ C-6), 16.9 ( 2 x C-26), 15.5 ( 2 x C-24), 15.3 ( 2 x C-25) ppm; MS (ESI, MeOH): $m / z=952.1(100 \%$, $\left.[\mathrm{M}+\mathrm{H}]^{+}\right), 974.0\left(22 \%,[\mathrm{M}+\mathrm{Na}]^{+}\right)$;

analysis calcd for $\mathrm{C}_{63} \mathrm{H}_{102} \mathrm{~N}_{2} \mathrm{O}_{4}$ (951.50): $\mathrm{C} 79.52$, H 10.81, N 2.94; found: C 79.35, H 11.03, N 2.77.

\section{$\mathrm{N}^{1}, \mathrm{~N}^{4}$-Bis-[(3ß)-3-hydroxy-olean-12-en-28-oyl]- 1,4-diaminobutane (17)}

Compound 17 (0.13 g, 83\%) showed: m.p. $186^{\circ} \mathrm{C}$; $\mathrm{R}_{F}=0.4$ (silica gel, toluene/ethyl acetate/formic $\mathrm{acid} /$ heptane, 80:26:5:1); $[\alpha]_{\mathrm{D}}=+47.00^{\circ}$ (c 0.31, $\left.\mathrm{CHCl}_{3}\right)$;

IR (KBr): $v=3390 b r, 2926 m, 2864 w, 1640 m, 1520 m$, $1463 m, 1386 w, 1260 s, 1092 s, 1026 s \mathrm{~cm}^{-1}$;

${ }^{1} \mathrm{H}$ NMR $\left(400 \mathrm{MHz}, \mathrm{CDCl}_{3}\right): \delta=6.06(t, J=5.6 \mathrm{~Hz}$, $2 \mathrm{H}, 2 \times \mathrm{NH}), 5.37(t, J=3.5 \mathrm{~Hz}, 2 \mathrm{H}, 2 \times 12-\mathrm{H}), 3.35$ $(d q, J=12.9,6.4 \mathrm{~Hz}, 2 \mathrm{H}, 2 \times 31-\mathrm{H}), 3.21(d d, J=$ 11.1, $4.6 \mathrm{~Hz}, 2 \mathrm{H}, 2 \times 3-\mathrm{H}), 3.04(d q, J=11.6,6.5 \mathrm{~Hz}$, $2 \mathrm{H},(2 \times 31-\mathrm{H}), 2.53(d d, J=12.9,4.1 \mathrm{~Hz}, 2 \mathrm{H}, 2 \mathrm{x}$ $18-\mathrm{H}), 2.01-1.92(m, 2 \mathrm{H}, 2 \times 16-\mathrm{H}), 1.95-1.87$ $(m, 2 \mathrm{H}, 2 \times 11-\mathrm{H}), 1.81-1.56(m, 22 \mathrm{H}, 2 \times 1-\mathrm{H}+2 \times$ $2-\mathrm{H}+2 \times 6-\mathrm{H}+2 \times 7-\mathrm{H}+2 \times 9-\mathrm{H}+2 \times 11-\mathrm{H}+2 \times$ $16-\mathrm{H}+2 \times 19-\mathrm{H}+2 \times 32-\mathrm{H}), 1.54(d t, J=13.9,3.4$ $\mathrm{Hz}, 2 \mathrm{H}, 2$ x $15-\mathrm{H}), 1.50-1.39(m, 2 \mathrm{H}, 2$ × 22-H), 1.39$1.27(m, 6 \mathrm{H}, 2 \times 6-\mathrm{H}+2 \times 7-\mathrm{H}+2 \times 21-\mathrm{H}), 1.27-1.22$ $(m, 2 \mathrm{H} 2 \times 22-\mathrm{H}), 1.22-1.17(m, 4 \mathrm{H}, 2 \times 19-\mathrm{H}+2 \times$ 21-H), 1.18-1.14 (s, 6H, 2 x 27-H), 1.07-0.99 ( $m, 4 \mathrm{H}$, $2 \times 1-\mathrm{H}+2 \times 15-\mathrm{H}), 0.99(s, 6 \mathrm{H}, 2 \times 23-\mathrm{H}), 0.91$ $(s, 6 \mathrm{H}, 2 \times 25-\mathrm{H}), 0.91(s, 6 \mathrm{H},(2 \times 30-\mathrm{H}), 0.90(s, 6 \mathrm{H}$, $2 \times 29-\mathrm{H}), 0.78(s, 6 \mathrm{H}, 2 \times 24-\mathrm{H}), 0.75(s, 6 \mathrm{H}, 2 \times 26-$ $\mathrm{H}), 0.71(s, 2 \mathrm{H}, 2 \times 5-\mathrm{H}) \mathrm{ppm}$;

${ }^{13} \mathrm{C} \mathrm{NMR}\left(100 \mathrm{MHz}, \mathrm{CDCl}_{3}\right): \delta=178.2(2 \times \mathrm{C}-28)$, 145.0 ( 2 x C-13), 122.7 ( 2 x C-12), 78.9 ( $2 \times$ C-3), 55.1 ( 2 x C-5), 47.5 ( 2 x C-9), 46.7 ( $2 \times$ C-17), 46.2 (2 x C-19), 42.1 (2 x C-18), 42.0 (2 x C-14), 39.3 (2 x C-8), 39.0 ( 2 x C-31), 38.7 ( 2 x C-1), 38.4 ( $2 \times$ C-4), 36.9 ( 2 x C-10), 34.1 ( 2 x C-21), 32.9 ( 2 x C-29), 32.5 (2 x C-7), 32.3 (2 x C-22), 30.7 (2 x C-20), 28.0 ( 2 x C-23), 27.3 (2 x C-32), 27.1 (2 x C-2), 26.9 ( 2 x C-15), 25.7 ( $2 \times \mathrm{C}-27), 23.7$ ( $2 \times \mathrm{C}-11), 23.6$ ( $2 \times$ C-30), 23.5 ( 2 x C-16), 18.2 ( 2 x C-6), 16.9 ( 2 x C-6), 15.5 (2 x C-24), 15.3 (2 x C-25) ppm; MS (ESI, $\mathrm{MeOH}): m / z=965.3\left(92 \%,[\mathrm{M}-\mathrm{H}]^{-}\right.$;

analysis calcd for $\mathrm{C}_{64} \mathrm{H}_{104} \mathrm{~N}_{2} \mathrm{O}_{4}$ (965.52): C 79.61, H 10.86, N 2.90; found: C 79.40, H 10.99, N 2.73. 


\section{$\mathrm{N}^{1}, \mathrm{~N}^{5}$-Bis-[(3ß)-3-hydroxy-olean-12-en-28-oyl]- 1,5-diaminopentane (18)}

Compound $18(0.04 \mathrm{~g}, 76 \%)$ showed: m.p. $182^{\circ} \mathrm{C}$; $\mathrm{R}_{F}=0.3$ (silica gel, toluene/ethyl acetate/formic acid/heptane, 80:26:5:1); $[\alpha]_{\mathrm{D}}=+35.37^{\circ}$ (c 0.36 , $\left.\mathrm{CHCl}_{3}\right)$;

IR $(\mathrm{KBr}): v=3387 b r, 2924 m, 2854 w, 1638 m, 1518 m$, $1455 m, 1363 m, 1037 m, 996 m \mathrm{~cm}^{-1}$;

${ }^{1} \mathrm{H}$ NMR $\left(500 \mathrm{MHz}, \mathrm{CDCl}_{3}\right): \delta=6.00(t, J=5.5 \mathrm{~Hz}$, $2 \mathrm{H}, 2 \times \mathrm{NH}), 5.31(t, J=3.5 \mathrm{~Hz}, 2 \mathrm{H}, 2 \times 12-\mathrm{H}), 3.31$ $(d q, J=13.4,6.8 \mathrm{~Hz}, 2 \mathrm{H}, 2 \times 31-\mathrm{H}), 3.25-3.18(d d, J$ $=11.2,4.6 \mathrm{~Hz}, 2 \mathrm{H}, 2 \times 3-\mathrm{H}), 3.01(d q, J=12.8,6.5$ $\mathrm{Hz}, 2 \mathrm{H},(2 \times 31-\mathrm{H}), 2.02-1.98$ ( $m, 2 \mathrm{H} 2 \times 16-\mathrm{H}), 1.98$ $1.94(m, 2 \mathrm{H}, 2 \mathrm{x} 11-\mathrm{H}), 1.93(d, J=3.3 \mathrm{~Hz}, 2 \mathrm{H}, 2 \times$ $18-\mathrm{H}), 1.87(d t, J=11.0,2.9 \mathrm{~Hz}, 2 \mathrm{H}, 2$ x $22-\mathrm{H}), 1.78$ $1.50(m, 20 \mathrm{H}, 2 \times 1-\mathrm{H}+2 \times 2-\mathrm{H}+2 \times 9-\mathrm{H}+2 \times$ $11-\mathrm{H}+2 \times 16-\mathrm{H}+2 \times 34-\mathrm{H}+2 \times 35-\mathrm{H}), 1.44(\mathrm{~m}, 12 \mathrm{H}$, $2 \times 15-\mathrm{H}+2 \times 7-\mathrm{H}+2 \times 6-\mathrm{H}+2 \times 21-\mathrm{H}+2 \times 22-\mathrm{H}$ $+(2 \times 19-\mathrm{H}), 1.40-1.25(m, 6 \mathrm{H}, 2 \times 6-\mathrm{H}+2 \times 7-\mathrm{H}+$ $2 \times 21-\mathrm{H}), 1.10(s, 6 \mathrm{H}, 2 \times 27-\mathrm{H}), 1.08-1.00(m, 4 \mathrm{H}$, $2 \times 1-\mathrm{H}+2 \times 15-\mathrm{H}), 0.99(s, 6 \mathrm{H},(2 \times 23-\mathrm{H}), 0.95$ $(s, 6 \mathrm{H}, 2 \times 30-\mathrm{H}), 0.92(s, 6 \mathrm{H}, 2 \times 25-\mathrm{H}), 0.87(s, 6 \mathrm{H}$, $2 \times 26-\mathrm{H}), 0.78(s, 6 \mathrm{H}, 2 \times 24-\mathrm{H}), 0.78(s, 6 \mathrm{H}, 2 \times$ 29-H), 0.71 ( $m, 2 \mathrm{H}, 2$ x 5-H) ppm;

${ }^{13} \mathrm{C} \mathrm{NMR}\left(125 \mathrm{MHz}, \mathrm{CDCl}_{3}\right): \delta=178.1(2 \times \mathrm{C}-28)$, 140.0 ( 2 x C-13), 125.5 ( 2 x C-12), 78.9 ( $2 \times$ C-3), 55.1 ( 2 x C-5), 53.9 ( 2 x C-18), 47.7 ( 2 x C-17), 47.5 ( 2 x C-9), 42.5 (2 x C-14), 39.7 (2 x C-8), 39.5 ( 2 x C-19), 39.2 ( 2 x C-31), 39.1 ( 2 x C-20), 38.7 ( $2 \times$ C-1), 38.6 ( 2 x C-4), 37.2 ( 2 x C-22), 36.9 ( 2 x C-10), 32.7 ( $2 \times$ C-7), 30.8 ( 2 x C-21), 28.1 ( 2 x C-23), 27.8 ( 2 x C-15), 27.1 ( 2 x C-32), 26.4 ( 2 x C-33), 24.8 ( 2 x C-2), 24.5 ( 2 x C-16), 23. ( $2 \times$ C-27), 23.2 ( $2 \times$ C-2), 21.2 ( 2 x C-30), 18.2 ( 2 x C-6), 17.2 ( 2 x C-24), 16.9 ( 2 x C-29), 15.6 ( 2 x C-26), 15.5 ( 2 x C-25) ppm; MS (ESI, MeOH): $m / z=994.7\left(100 \%,[\mathrm{M}+\mathrm{H}]^{+}\right)$, $1017.6\left(90 \%,[\mathrm{M}+\mathrm{Na}]^{+}\right)$;

analysis calcd for $\mathrm{C}_{65} \mathrm{H}_{106} \mathrm{~N}_{2} \mathrm{O}_{4}$ (979.55): C 79.70, H 10.91, N 2.86; found: C 79.51, H 11.13, N 2.64.

\section{$\mathbf{N}^{1}, \mathbf{N}^{6}$-Bis-[(3ß)-3-hydroxy-urs-12-en-28-oyl]-1,6- diaminohexane (19)}

Compound $19(0.18 \mathrm{~g}, 76 \%)$ showed: m.p. $183^{\circ} \mathrm{C}$ (lit.: 191-193 ${ }^{\circ} \mathrm{C}$ ); $\mathrm{R}_{F}=0.3$ (silica gel, toluene/ethyl acetate/formic acid/heptane, 80:26:5:1); $[\alpha]_{\mathrm{D}}=$ $+50.40^{\circ}\left(c 0.33, \mathrm{CHCl}_{3}\right)$;

IR $(\mathrm{KBr}): v=3404 b r, 2927 s, 2863 m, 1639 s, 1521 s$, $1463 s, 1386 s, 1242 m, 1030 s, 996 s \mathrm{~cm}^{-1}$;

${ }^{1} \mathrm{H} \mathrm{NMR}\left(400 \mathrm{MHz}, \mathrm{CDCl}_{3}\right): \delta=5.98-5.93(m, 2 \mathrm{H}, 2$ x NH), $5.37(t, J=3.5 \mathrm{~Hz}, 2 \mathrm{H}, 2$ x $12-\mathrm{H}), 3.35(d q, J$ $=13.7,7.0 \mathrm{~Hz}, 2 \mathrm{H}, 2 \times 31-\mathrm{H}), 3.22(d d, J=11.2,4.5$ $\mathrm{Hz}, 2 \mathrm{H}, 2 \times 3-\mathrm{H}), 3.03-2.93$ ( $m, 2 \mathrm{H}, 2 \times 31-\mathrm{H}), 2.50$ $(d d, J=12.8,3.8 \mathrm{~Hz}, 2 \mathrm{H}, 2 \times 18-\mathrm{H}), 1.97(d d, J=$ $13.6,3.8 \mathrm{~Hz}, 2 \mathrm{H}, 2 \times 16-\mathrm{H}), 1.91(m, 2 \mathrm{H}, 2 \times 11-\mathrm{H})$, $1.81-1.57(m, 28 \mathrm{H}, 2 \times 1-\mathrm{H}+2 \times 2-\mathrm{H}+2 \times 6-\mathrm{H}+2 \times$ $7-\mathrm{H}+2 \times 9-\mathrm{H}+2 \times 11-\mathrm{H}+2 \times 16-\mathrm{H}+2 \times 19-\mathrm{H}+2 \times$ $32-\mathrm{H}+2 \times 33-\mathrm{H}), 1.57-1.51(m, 2 \mathrm{H}, 2 \times 15-\mathrm{H}), 1.50$ $1.40(m, 2 \mathrm{H}, 2 \times 22-\mathrm{H}), 1.40-1.27(m, 6 \mathrm{H}, 2 \times 6-\mathrm{H}+$ $2 \times 7-\mathrm{H}+2 \times 21-\mathrm{H}), 1.25(m, 2 \mathrm{H} 2 \times 22-\mathrm{H}), 1.19$ $(\mathrm{d}, J=11.3 \mathrm{~Hz}, 4 \mathrm{H}, 2 \times 19-\mathrm{H}+2 \times 21-\mathrm{H}), 1.16(s, 6 \mathrm{H}$, $2 \times 27-\mathrm{H}), 1.07-1.00(m, 4 \mathrm{H}, 2 \times 1-\mathrm{H}+2 \times 15-\mathrm{H})$, $0.99(s, 6 \mathrm{H}, 2 \times 23-\mathrm{H}), 0.91(s, 6 \mathrm{H}, 2 \times 25-\mathrm{H}), 0.91$ $(s, 6 \mathrm{H},(2 \times 30-\mathrm{H}), 0.90(s, 6 \mathrm{H}, 2 \times 29-\mathrm{H}), 0.78(s, 6 \mathrm{H}$, $2 \times 24-\mathrm{H}), 0.76(s, 6 \mathrm{H}, 2 \times 26-\mathrm{H}), 0.72(s, 2 \mathrm{H}, 2 \times$ 5-H) ppm;

${ }^{13} \mathrm{C}$ NMR (100 MHz, $\left.\mathrm{CDCl}_{3}\right): \delta=178.1(2 \times \mathrm{C}-28)$, 145.1 ( $2 \times \mathrm{C}-13), 122.6$ ( $2 \times \mathrm{C}-12), 78.9(2 \times \mathrm{C}-3)$, 55.1 ( 2 x C-5), 47.5 ( 2 x C-9), 46.7 ( $2 \times \mathrm{C}-17), 46.2$ ( 2 x C-19), 42.3 (2 x C-18), 42.1 ( 2 x C-14), 39.3 ( 2 x C-8), 39.2 (2 x C-31), 38.7 ( 2 x C-1), 38.4 ( 2 x C-4) 36.9 ( 2 x C-10), 34.1 ( 2 × C-21), 32.9 ( 2 x C-29), 32.4 (2 x C-7), 32.4 (2 x C-22), 30.7 (2 x C-20), 29.3 ( 2 x C-32), 28.0 ( $2 \times$ C-23), 27.2 ( $2 \times$ C-2), $27.1(2 \times$ C-15) 26.6 (2 x C-33), 25.7 ( 2 x C-27), 23.7 ( 2 x C-11), 23.6 ( $2 \times \mathrm{C}-30), 23.5$ ( $2 \times \mathrm{C}-16), 18.2(2 \times$ C-6), 16.9 ( 2 x C-6), 15.5 ( 2 x C-24), 15.3 ( 2 x C-25) ppm;

MS (ESI, $\mathrm{MeOH}): m / z=994.6\left(90 \%,[\mathrm{M}+\mathrm{H}]^{+}\right)$, $1016.6\left(100 \%,[\mathrm{M}+\mathrm{Na}]^{+}\right)$;

analysis calcd for C66H108N2O4 (993.58): C 79.78, H 10.96, N 2.82; found: C 79.62, H 11.07, N 2.69.

\section{$\mathbf{N}^{1}, \mathbf{N}^{7}$-Bis-[(3ß)-3-hydroxy-olean-12-en-28-oyl]- 1,7-diaminoheptane (20)}

Compound $20\left(0.12\right.$ g, 87\%) showed: m.p. $183^{\circ} \mathrm{C}$; $\mathrm{R}_{F}=0.3$ (silica gel, toluene/ethyl acetate/formic $\mathrm{acid} /$ heptane, 80:26:5:1); $[\alpha]_{\mathrm{D}}=+45.85^{\circ}$ (c 0.36 , $\left.\mathrm{CHCl}_{3}\right)$;

IR $(\mathrm{KBr}): v=3407 \mathrm{br}, 2927 \mathrm{~s}, 2861 \mathrm{~m}, 1641 \mathrm{~s}, 1519 m$, $1462 \mathrm{~s}, 1386 \mathrm{~m}, 1363 \mathrm{~m}, 1212 \mathrm{~m}, 1030 \mathrm{~m}, 996 \mathrm{~m} \mathrm{~cm}^{-1}$;

${ }^{1} \mathrm{H}$ NMR $\left(400 \mathrm{MHz}, \mathrm{CDCl}_{3}\right): \delta=5.93-5.88(m, 2 \mathrm{H}$, $2 \times \mathrm{NH}), 5.37(t, J=3.5 \mathrm{~Hz}, 2 \mathrm{H}, 2 \times 12-\mathrm{H}), 3.34(d q$, $J=13.7,7.1 \mathrm{~Hz}, 2 \mathrm{H},(2 \times 31-\mathrm{H}), 3.21(d d, J=11.2$, $4.5 \mathrm{~Hz}, 2 \mathrm{H}, 2 \times 3-\mathrm{H}), 3.03-2.93(m, 2 \mathrm{H},(2 \times 31-\mathrm{H})$, $2.49(d d, J=12.8,3.6 \mathrm{~Hz}, 2 \mathrm{H}, 2 \times 18-\mathrm{H}), 1.97(d d, J$ $=13.7,3.7 \mathrm{~Hz}, 2 \mathrm{H}, 2 \times 16-\mathrm{H}), 1.91(d d, J=8.9,3.3$ $\mathrm{Hz}, 2 \mathrm{H}, 2 \times 11-\mathrm{H}), 1.81-1.56(m, 30 \mathrm{H}, 2 \times 1-\mathrm{H}+2 \times$ $2-\mathrm{H}+2 \times 6-\mathrm{H}+2 \times 7-\mathrm{H}+2 \times 9-\mathrm{H}+2 \times 11-\mathrm{H}+2 \times$ $16-\mathrm{H}+2 \times 19-\mathrm{H}+2 \times 32-\mathrm{H}+2 \times 33-\mathrm{H}+2 \times 34-\mathrm{H})$, $1.54(d d, J=9.7,4.3 \mathrm{~Hz}, 2 \mathrm{H}, 2 \times 15-\mathrm{H}), 1.50-1.40(\mathrm{~m}$, $2 \mathrm{H}, 2 \times 22-\mathrm{H}), 1.40-1.27(m, 6 \mathrm{H}, 2 \times 6-\mathrm{H}+2 \times 7-\mathrm{H}+$ $2 \times 21-\mathrm{H}), 1.25(m, 2 \mathrm{H} 2 \times 22-\mathrm{H}), 1.19(d, J=11.3$ $\mathrm{Hz}, 4 \mathrm{H}, 2 \times 19-\mathrm{H}+2 \times 21-\mathrm{H}), 1.16(s, 6 \mathrm{H}, 2 \times 27-\mathrm{H})$, $1.03(m, 4 \mathrm{H}, 2 \times 1-\mathrm{H}+2 \times 15-\mathrm{H}), 0.99(s, 6 \mathrm{H}, 2 \times 23-$ $\mathrm{H}), 0.91(s, 6 \mathrm{H}, 2 \times 25-\mathrm{H}), 0.91(s, 6 \mathrm{H},(2 \times 30-\mathrm{H})$, $0.90(s, 6 \mathrm{H}, 2 \times 29-\mathrm{H}), 0.78(s, 6 \mathrm{H}, 2 \times 24-\mathrm{H}), 0.76$ $(s, 6 \mathrm{H}, 2 \times 26-\mathrm{H}), 0.72(s, 2 \mathrm{H}, 2 \times 5-\mathrm{H}) \mathrm{ppm}$;

${ }^{13} \mathrm{C}$ NMR $\left(100 \mathrm{MHz}, \mathrm{CDCl}_{3}\right): \delta=178.1(2 \times \mathrm{C}-28)$, 145.1 ( 2 x C-13), 122.6 ( 2 x C-12), 78.9 ( $2 \times$ C-3), 55.1 ( 2 x C-5), 47.5 ( $2 \times$ C-9), 46.8 ( $2 \times \mathrm{C}-17), 46.2$ (2 x C-19), 42.3 (2 x C-18), 42.0 ( 2 x C-14), 39.4 ( 2 x C-8), 39.3 (2 x C-31), 38.7 ( 2 x C-1), 38.4 (2 x C-4), 36.9 ( 2 x C-10), 34.1 ( 2 x C-21), 32.9 ( 2 x C-29), 32.5 ( 2 x C-7), 32.3 ( 2 x C-22), 30.7 ( $2 \times$ C-20), $29.3(2 \times$ C-32), 28.9 (C-34), 28.0 ( $2 \times \mathrm{C}-23), 27.2$ ( $2 \times \mathrm{C}-2)$, 27.1 ( 2 x C-15), 27.0 ( 2 x C-33), 25.7 ( 2 x C-27), 23.8 ( 2 x C-11), 23.5 (2 x C-30), 23.5 ( 2 x C-16), $18.2(2 \times$ C-6), 16.9 ( 2 x C-6), 15.5 (2 x C-24), 15.3 (2 x C-25) ppm;

MS (ESI, MeOH): $m / z=1008.8\left(14 \%,[\mathrm{M}+\mathrm{H}]^{+}\right)$, $1031.7\left(43 \%,[\mathrm{M}+\mathrm{Na}]^{+}\right)$; 
analysis calcd for $\mathrm{C}_{67} \mathrm{H}_{110} \mathrm{~N}_{2} \mathrm{O}_{4}$ (xx): C 79.86, H 11.00, N 2.78; found: C 79.68, H 11.18, N 2.69.

\section{$\mathrm{N}^{1}, \mathrm{~N}^{2}$-Bis-[(3ß)-3-hydroxy-urs-12-en-28-oyl]-1,2- diaminoethane (21)}

Compound 21 ( 0.18 g, 85\%) showed: m.p. $228^{\circ} \mathrm{C}$; $\mathrm{R}_{F}=0.3$ (silica gel, toluene/ethyl acetate/formic $\mathrm{acid} / \mathrm{heptane}, 80: 26: 5: 1) ;[\alpha]_{\mathrm{D}}=+44.32^{\circ}$ (c 0.31 , $\left.\mathrm{CHCl}_{3}\right)$;

IR $(\mathrm{KBr}): v=3383 \mathrm{br}, 2927 \mathrm{~s}, 2870 m, 1632 \mathrm{~s}, 1533 m$, $1455 \mathrm{~m}, 1378 w, 1280 w, 1089 w, 1030 \mathrm{~m}, 995 \mathrm{~m} \mathrm{~cm}^{-1}$;

${ }^{1} \mathrm{H}$ NMR $\left(400 \mathrm{MHz}, \mathrm{CDCl}_{3}\right): \delta=6.53(\mathrm{~m}, 2 \mathrm{H}, 2 \mathrm{x}$ $\mathrm{NH}), 5.36(t, J=3.4 \mathrm{~Hz}, 2 \mathrm{H}, 2 \times 12-\mathrm{H}), 3.48(d, J=$ $9.4 \mathrm{~Hz}, 2 \mathrm{H}, 2 \mathrm{x} 31-\mathrm{H}), 3.21(d d, J=11.2,4.6 \mathrm{~Hz}, 2 \mathrm{H}$, $2 \times 3-\mathrm{H}), 3.09(d, J=9.0 \mathrm{~Hz}, 2 \mathrm{H},(2 \times 31-\mathrm{H}), 2.02$ $(d, J=4.1 \mathrm{~Hz}, 2 \mathrm{H} 2 \times 16-\mathrm{H}), 2.00-1.94(m, 2 \mathrm{H}, 2 \times$ $11-\mathrm{H}), 1.91(m, 2 \mathrm{H}, 2 \times 18-\mathrm{H}), 1.89-1.84(m, 2 \mathrm{H}, 2 \times$ $22-\mathrm{H}), 1.76-1.51(m, 12 \mathrm{H}, 2 \times 1-\mathrm{H}+2 \times 2-\mathrm{H}+2 \times$ $9-\mathrm{H}+2 \times 11-\mathrm{H}+2 \times 16-\mathrm{H}), 1.50-1.48(\mathrm{~m}, 6 \mathrm{H}, 2 \times$ $15-\mathrm{H}+2 \times 7-\mathrm{H}+2 \times 6-\mathrm{H}), 1.47(m, 6 \mathrm{H}, 2 \times 21-\mathrm{H}+2$ x $22-\mathrm{H}+2$ x 19-H), 1.40-1.25 (m, 6H, 2 x 6-H + 2 x $7-\mathrm{H}+2 \times 21-\mathrm{H}), 1.09(s, 6 \mathrm{H}, 2 \times 27-\mathrm{H}), 1.08-1.02$ $(m, 4 \mathrm{H}, 2 \times 1-\mathrm{H}+2 \times 15-\mathrm{H}), 0.99(s, 6 \mathrm{H}, 2 \times 23-\mathrm{H})$, $0.95(s, 6 \mathrm{H}, 2 \times 30-\mathrm{H}), 0.92(s, 6 \mathrm{H}, 2 \times 25-\mathrm{H}), 0.87$ $(s, 6 \mathrm{H}, 2 \times 26-\mathrm{H}), 0.78(s, 6 \mathrm{H}, 2 \times 24-\mathrm{H}), 0.75(s, 6 \mathrm{H}$, $2 \times 29-\mathrm{H}), 0.72(m, 2 \mathrm{H}, 2 \times 5-\mathrm{H}) \mathrm{ppm}$;

${ }^{13} \mathrm{C} \mathrm{NMR}\left(100 \mathrm{MHz}, \mathrm{CDCl}_{3}\right): \delta=178.1(2 \times \mathrm{C}-28)$, 139.2 ( $2 \times \mathrm{C}-13), 125.9$ ( $2 \times \mathrm{C}-12), 78.9(2 \times \mathrm{C}-3)$, 55.1 ( 2 x C-5), 53.3 ( 2 x C-18), 47.6 ( 2 x C-17), 47.5 ( 2 x C-9), 42.3 ( 2 x C-14), 39.7 ( 2 x C-8), 39.9 ( 2 x C-31), 39.5 ( 2 x C-19), 39.0 ( 2 x C-20), 38.7 ( 2 x C-1), 38.5 ( 2 x C-4), 37.4 ( $2 \times$ C-22), 36.9 ( 2 x C-10), 32.6 ( $2 \times \mathrm{C}-7), 30.8$ ( $2 \times \mathrm{C}-21), 28.1$ ( $2 \times \mathrm{C}-23), 27.8$ ( 2 x C-15), 27.1 ( 2 x C-2), 24.7 ( 2 x C-16), 23.5 ( 2 x C-27), 23.3 ( $2 \times \mathrm{C}-11), 21.2$ ( 2 x C-30), $18.1(2 \times$ C-6), 17.2 ( 2 x C-24), 16.8 ( $2 \times$ C-29), 16.7 ( $2 \times$ $\mathrm{C}-26), 15.5$ (2 x C-25) ppm;

MS (ESI, MeOH): $m / z=938.5\left(60 \%,[\mathrm{M}+\mathrm{H}]^{+}\right), 960.5$ $\left(100 \%,[\mathrm{M}+\mathrm{Na}]^{+}\right)$;

analysis calcd for $\mathrm{C}_{62} \mathrm{H}_{100} \mathrm{~N}_{2} \mathrm{O}_{4}$ (937.49): C 79.43 , H 10.75, N 2.99; found: C 70.23, H 10.95, N 2.71.

\section{$\mathbf{N}^{1}, N^{3}$-Bis-[(3ß)-3-hydroxy-urs-12-en-28-oyl]-1,3- diaminopropane (22)}

Compound $22\left(0.02\right.$ g, 56\%) showed: m.p. $242^{\circ} \mathrm{C}$ $\mathrm{R}_{F}=0.3$ (silica gel, toluene/ethyl acetate/formic $\mathrm{acid} / \mathrm{heptane}, 80: 26: 5: 1) ;[\alpha]_{\mathrm{D}}=+20.84^{\circ}$ (c 0.3 , $\left.\mathrm{CHCl}_{3}\right)$;

IR $(\mathrm{KBr}): v=3383 \mathrm{br}, 2927 \mathrm{~s}, 2870 \mathrm{~m}, 1632 \mathrm{~m}, 1532 \mathrm{~m}$, $1454 m, 1378 m, 1259 s, 1088 m, 1029 m, 996 w \mathrm{~cm}^{-1}$; ${ }^{1} \mathrm{H}$ NMR $\left(500 \mathrm{MHz}, \mathrm{CDCl}_{3}\right): \delta=6.42(m, 2 \mathrm{H}, 2 \mathrm{x}$ $\mathrm{NH}), 5.36(t, J=3.5 \mathrm{~Hz}, 2 \mathrm{H}, 2 \mathrm{x} 12-\mathrm{H}), 3.31-3-22(d$, $J=9.4 \mathrm{~Hz}, 2 \mathrm{H},(2 \times 31-\mathrm{H}), 3.20(m, 2 \mathrm{H}, 2 \times 3-\mathrm{H}), 3.10$ $(d d, J=13.4,5.9 \mathrm{~Hz}, 2 \mathrm{H}, 2 \times 31-\mathrm{H}), 2.03(d, J=4.2$ $\mathrm{Hz}, 2 \mathrm{H} 2 \times 16-\mathrm{H}), 2.01-1.94(m, 2 \mathrm{H}, 2 \times 11-\mathrm{H}), 1.92$ $(m, 2 \mathrm{H}, 2 \times 18-\mathrm{H}), 1.90-1.82(m, 2 \mathrm{H}, 2 \times 22-\mathrm{H}), 1.79$ $1.50(m, 14 \mathrm{H}, 2 \times 1-\mathrm{H}+2 \times 2-\mathrm{H}+2 \times 9-\mathrm{H}+2 \times$ $11-\mathrm{H}+2 \times 16-\mathrm{H}+2 \times 34-\mathrm{H}), 1.49(m, 6 \mathrm{H}, 2 \times 15-\mathrm{H}+$ $2 \times 7-\mathrm{H}+2 \times 6-\mathrm{H}), 1.48-1.44(m, 6 \mathrm{H}, 2 \times 21-\mathrm{H}+2 \times$ $22-\mathrm{H}+2 \times 19-\mathrm{H}), 1.43-1.24(m, 6 \mathrm{H}, 2 \times 6-\mathrm{H}+2 \times$ $7-\mathrm{H}+2 \times 21-\mathrm{H}), 1.10(s, 6 \mathrm{H}, 2 \times 27-\mathrm{H}), 1.08-1.00$ $(m, 4 \mathrm{H}, 2 \times 1-\mathrm{H}+2 \times 15-\mathrm{H}), 0.99(s, 6 \mathrm{H}, 2 \times 23-\mathrm{H})$, $0.94(s, 6 \mathrm{H}, 2 \times 30-\mathrm{H}), 0.91(s, 6 \mathrm{H}, 2 \times 25-\mathrm{H}), 0.87$ $(s, 6 \mathrm{H}, 2 \times 26-\mathrm{H}), 0.78(s, 6 \mathrm{H}, 2 \times 24-\mathrm{H}), 0.76(s, 6 \mathrm{H}$, $2 \times 29-\mathrm{H}), 0.71(\mathrm{~m}, 2 \mathrm{H}, 2 \times 5-\mathrm{H}) \mathrm{ppm}$;

${ }^{13} \mathrm{C} \mathrm{NMR}\left(125 \mathrm{MHz}, \mathrm{CDCl}_{3}\right): \delta=178.1(2 \times \mathrm{C}-28)$, 139.3 ( 2 x C-13), 125.7 ( 2 x C-12), 78.9 ( $2 \times$ C-3), 55.1 ( 2 x C-5), 53.5 ( 2 x C-18), 47.7 ( 2 x C-17), 47.5 (2 x C-9), 42.4 ( 2 x C-14), 39.7 ( 2 x C-8), 39.5 ( 2 x C-19), 39.0 (2 x C-20), 38.7 (2 x C-1), 38.6 ( 2 x C-4), 37.4 ( 2 x C-22), 36.9 ( 2 x C-10), 36.4 ( 2 x C-31), 32.8 ( 2 x C-7), 30.9 ( $2 \times$ C-21), 28.1 ( $2 \times$ C-23), 27.8 ( $2 \times$ C-15), 27.1 ( $2 \times$ C-2), 27.2 (2 x C-32), $24.7(2 \times$ C-16), 23.4 (2 x C-27), 23.3 (2 x C-2), 21.2 (2 x C-30), 18.2 ( 2 x C-6), 17.2 ( 2 x C-24), 17.0 ( 2 x C-29), 15.5 ( 2 x C-26), 15.5 ( 2 x C-25) ppm; MS (ESI, $\mathrm{MeOH}): m / z=951.9\left(40 \%,[\mathrm{M}+\mathrm{H}]^{+}\right), 973.8(8 \%$, $\left.[\mathrm{M}+\mathrm{Na}]^{+}\right)$

analysis calcd for $\mathrm{C}_{63} \mathrm{H}_{102} \mathrm{~N}_{2} \mathrm{O}_{4}$ (951.49): C 79.52, H 10.81, N 2.94; found: C 79.32, H 11.03, N 2.76.

\section{$\mathrm{N}^{1}, \mathrm{~N}^{4}$-Bis-[(3ß)-3-hydroxy-urs-12-en-28-oyl]-1,4- diaminobutane (23)}

Compound 23 (0.03 g, 54\%) showed: m.p. $245^{\circ} \mathrm{C} ; \mathrm{R}_{F}$ $=0.3$ (silica gel, toluene/ethyl acetate/formic $\mathrm{acid} /$ heptane, 80:26:5:1); $[\alpha]_{\mathrm{D}}=+33.23^{\circ}$ (c 0.16 , $\mathrm{CHCl}_{3}$ );

IR (KBr): $v=3422 b r, 2926 s, 2870 m, 1638 s, 1517 m$, $1454 \mathrm{~s}, 1378 \mathrm{~m}, 1243 w, 1045 \mathrm{~m}, 1029 \mathrm{~m}, 997 \mathrm{~m} \mathrm{~cm}^{-1}$;

${ }^{1} \mathrm{H}$ NMR $\left(400 \mathrm{MHz}, \mathrm{CDCl}_{3}\right): \delta=5.97(m, 2 \mathrm{H}, 2 \mathrm{x}$ $\mathrm{NH}), 5.31(t, J=3.6 \mathrm{~Hz}, 2 \mathrm{H}, 2 \times 12-\mathrm{H}), 3.40-3.29$ $(m, 2 \mathrm{H}, 2 \times 31-\mathrm{H}), 3.22(d t, J=10.8,5.3 \mathrm{~Hz}, 2 \mathrm{H}, 2 \mathrm{x}$ $3-\mathrm{H}), 2.99(d d, J=12.2,5.8 \mathrm{~Hz}, 2 \mathrm{H}, 2 \times 31-\mathrm{H}), 2.00$ $(t, J=2.3 \mathrm{~Hz}, 2 \mathrm{H} 2 \times 16-\mathrm{H}), 1.95(m, 2 \mathrm{H}, 2 \times 11-\mathrm{H})$, $1.88(d, J=3.2 \mathrm{~Hz}, 2 \mathrm{H}, 2 \times 18-\mathrm{H}), 1.85(d, J=3.1 \mathrm{~Hz}$, $2 \mathrm{H}, 2$ × $22-\mathrm{H}), 1.77-1.50(m, 16 \mathrm{H}, 2 \times 1-\mathrm{H}+2 \times 2-\mathrm{H}$ $+2 \times 9-\mathrm{H}+2 \times 11-\mathrm{H}+2 \times 16-\mathrm{H}+2 \times 34-\mathrm{H}), 1.45(\mathrm{~m}$, $12 \mathrm{H}, 2 \times 15-\mathrm{H}+2 \times 7-\mathrm{H}+2 \times 6-\mathrm{H}+2 \times 21-\mathrm{H}+2 \times$ $22-\mathrm{H}+2 \times 19-\mathrm{H}), 1.41-1.24(m, 6 \mathrm{H}, 2 \times 6-\mathrm{H}+2 \times$ $7-\mathrm{H}+2 \times 21-\mathrm{H}), 1.10(s, 6 \mathrm{H}, 2 \times 27-\mathrm{H}), 1.08-1.00$ $(m, 4 \mathrm{H}, 2 \times 1-\mathrm{H}+2 \times 15-\mathrm{H}), 0.99(s, 6 \mathrm{H}, 2 \times 23-\mathrm{H})$, $0.95(s, 6 \mathrm{H}, 2 \times 30-\mathrm{H}), 0.92(s, 6 \mathrm{H}, 2 \times 25-\mathrm{H}), 0.87$ $(s, 6 \mathrm{H}, 2 \times 26-\mathrm{H}), 0.78(s, 6 \mathrm{H}, 2 \times 24-\mathrm{H}), 0.77(s, 6 \mathrm{H}$, $2 \times 29-\mathrm{H}), 0.72(\mathrm{~m}, 2 \mathrm{H}, 2 \times 5-\mathrm{H}) \mathrm{ppm}$;

${ }^{13} \mathrm{C}$ NMR $\left(100 \mathrm{MHz}, \mathrm{CDCl}_{3}\right): \delta=178.1(2 \times \mathrm{C}-28)$, $140.0(2 \times \mathrm{C}-13), 125.5(2 \times \mathrm{C}-12), 78.9(2 \times \mathrm{C}-3)$, 55.1 ( 2 x C-5), 53.8 ( 2 x C-18), 47.6 ( 2 x C-17), 47.5 (2 x C-9), 42.5 (2 x C-14), 39.7 (2 x C-8), 39.5 (2 x C-19), 39.1 ( 2 x C-20), 38.9 ( 2 x C-31), 38.7 ( 2 x C-1), 38.6 ( 2 x C-4), 37.2 ( 2 x C-22), 36.9 ( 2 x C-10), 32.7 ( 2 x C-7), 30.9 ( 2 x C-21), 28.1 ( 2 x C-23), 27.8 ( $2 \times \mathrm{C}-15), 27.1$ ( $2 \times \mathrm{C}-32), 26.9(2 \times \mathrm{C}-2), 24.7(2 \times$ C-16), 23.4 ( $2 \times \mathrm{C}-27), 23.2(2 \times \mathrm{C}-2), 21.2(2 \times$ C-30), 18.2 ( $2 \times \mathrm{C}-6), 17.2$ (2 x C-24), $17.0(2 \times$ C-29), 15.5 ( 2 x C-26), 15.5 ( 2 × C-25) ppm; MS (ESI, $\mathrm{MeOH}): m / z=967.5\left(60 \%,[\mathrm{M}+\mathrm{H}]^{+}\right), 990.5(100 \%$, $\left.[\mathrm{M}+\mathrm{Na}]^{+}\right)$

analysis calcd for $\mathrm{C}_{64} \mathrm{H}_{104} \mathrm{~N}_{2} \mathrm{O}_{4}$ (965.52): C 79.61, H 10.86, N 2.90; found: C 79.45, H 11.03, N 2.84 . 


\section{$\mathbf{N}^{1}, N^{5}$-Bis-[(3ß)-3-hydroxy-urs-12-en-28-oyl]-1,5- diaminopentane (24)}

Compound $24(0.1 \mathrm{~g}, 77 \%)$ showed: m.p. $158^{\circ} \mathrm{C}$; $\mathrm{R}_{F}=0.3$ (silica gel, toluene/ethyl acetate/formic acid/heptane, 80:26:5:1); $[\alpha]_{\mathrm{D}}=+42.57^{\circ}$ (c 0.31 , $\left.\mathrm{CHCl}_{3}\right)$;

IR (KBr): $v=3396 b r, 2924 m, 2869 w, 1641 m, 1520 w$, $1455 w, 1386 w, 1260 \mathrm{~m}, 1091 \mathrm{~s}, 1026 \mathrm{sm}^{-1}$;

${ }^{1} \mathrm{H}$ NMR (400 MHz, $\left.\mathrm{CDCl}_{3}\right): \delta=5.92(t, J=5.6 \mathrm{~Hz}$, $2 \mathrm{H}, 2 \times \mathrm{NH}), 5.31(t, J=3.2 \mathrm{~Hz}, 2 \mathrm{H}, 2 \times 12-\mathrm{H}), 3.3$ $(d q, J=13.5,6.9 \mathrm{~Hz}, 2 \mathrm{H}, 2 \times 31-\mathrm{H}), 3.25-3.18(\mathrm{~m}$, $2 \mathrm{H}, 2 \times 3-\mathrm{H}), 3.03-2.92(m, 2 \mathrm{H}, 2 \times 31-\mathrm{H}), 2.00(d, J$ $=4.2 \mathrm{~Hz}, 2 \mathrm{H} 2 \times 16-\mathrm{H}), 1.98-1.94(m, 2 \mathrm{H}, 2 \times 11-\mathrm{H})$, $1.83(d, J=2.3 \mathrm{~Hz}, 2 \mathrm{H}, 2 \times 18-\mathrm{H}), 1.91-1.82(m, 2 \mathrm{H}$, $2 \times 22-\mathrm{H}), 1.78-1.50(m, 18 \mathrm{H}, 2 \times 1-\mathrm{H}+2 \times 2-\mathrm{H}+$ $2 \times 9-\mathrm{H}+2 \times 11-\mathrm{H}+2 \times 16-\mathrm{H}+2 \times 34-\mathrm{H}+35-\mathrm{H})$, $1.49-1.42(m, 12 \mathrm{H}, 2 \times 15-\mathrm{H}+2 \times 7-\mathrm{H}+2 \times 6-\mathrm{H}+2$ x 21-H + 2 x 22-H + (2 x 19-H), 1.41-1.26 (m, 6H, 2 x $6-\mathrm{H}+2 \times 7-\mathrm{H}+2 \times 21-\mathrm{H}), 1.09(s, 6 \mathrm{H}, 2 \times 27-\mathrm{H})$, $1.08-1.00(m, 4 \mathrm{H}, 2 \times 1-\mathrm{H}+2 \times 15-\mathrm{H}), 0.99(s, 6 \mathrm{H}$, $(2 \times 23-\mathrm{H}), 0.95(s, 6 \mathrm{H}, 2 \times 30-\mathrm{H}), 0.92(s, 6 \mathrm{H}, 2 \times$ $25-\mathrm{H}), 0.87$ ( $s, 6 \mathrm{H}, 2 \times 26-\mathrm{H}), 0.78(s, 6 \mathrm{H}, 2 \times 24-\mathrm{H})$, $0.77(s, 6 \mathrm{H}, 2 \times 29-\mathrm{H}), 0.71(\mathrm{~m}, 2 \mathrm{H}, 2 \times 5-\mathrm{H}) \mathrm{ppm}$;

$\left.{ }^{13} \mathrm{C} \mathrm{NMR} \mathrm{(100} \mathrm{MHz,} \mathrm{CDCl}_{3}\right): \delta=178.1$ (2 x C-28), 140.0 ( $2 \times \mathrm{C}-13), 125.5$ ( $2 \times \mathrm{C}-12), 78.9(2 \times \mathrm{C}-3)$, 55.2 ( $2 \times$ C-5), 53.9 ( 2 x C-18), 47.6 ( 2 x C-17), 47.5 ( 2 x C-9), 42.5 ( 2 x C-14), 39.7 ( 2 x C-8), 39.5 ( 2 x C-19), 39.3 ( 2 x C-31), 39.1 ( 2 x C-20), 38.7 ( 2 x C-1), 38.6 ( 2 x C-4), 37.2 ( 2 x C-22), 36.9 ( 2 x C-10), 32.7 ( 2 x C-7), 30.8 ( 2 x C-21), 29.0 (C-33), 28.1 ( 2 x C-23), 27.8 ( $2 \times$ C-15), 27.1 ( 2 x C-32), 24.8 ( $2 \times$ C-2), 24.5 (2 x C-16), 23.4 (2 x C-27), 23.2 ( 2 x C-2), 21.2 ( $2 \times$ C-30), 18.2 ( $2 \times$ C-6), 17.2 ( 2 x C-24), 16.9 (2 x C-29), 15.6 (2 x C-26), 15.5 ( 2 x C-25) ppm; MS (ESI, MeOH): $m / z=980.3\left(20 \%,[\mathrm{M}+\mathrm{H}]^{+}\right)$, $1002.3\left(100 \%,[\mathrm{M}+\mathrm{Na}]^{+}\right)$;

analysis calcd for $\mathrm{C}_{65} \mathrm{H}_{106} \mathrm{~N}_{2} \mathrm{O}_{4}$ (979.55): C 79.50, H 10.91, N 2.86; found: C 79.36, H 11.13, N 2.67.

\section{$\mathbf{N}^{1}, \mathbf{N}^{6}$-Bis-[(3ß)-3-hydroxy-urs-12-en-28-oyl]-1,6- diaminohexane (25)}

Compound 25 (0.11 g, 70\%) showed: m.p. $182^{\circ} \mathrm{C}$; $\mathrm{R}_{F}=0.3$ (silica gel, toluene/ethyl acetate/formic acid/heptane, 80:26:5:1); $[\alpha]_{\mathrm{D}}=+35.37^{\circ}$ (c 0.36 , $\left.\mathrm{CHCl}_{3}\right)$;

IR $(\mathrm{KBr}): v=3387 \mathrm{br}, 2924 m, 2854 w, 1638 m, 1518 m$, $1455 \mathrm{~m}, 1363 \mathrm{~m}, 1037 \mathrm{~m}, 996 \mathrm{~m} \mathrm{~cm}^{-1}$;

${ }^{1} \mathrm{H}$ NMR $\left(500 \mathrm{MHz}, \mathrm{CDCl}_{3}\right): \delta=6.00(t, J=5.5 \mathrm{~Hz}$, $2 \mathrm{H}, 2 \times \mathrm{NH}), 5.31(t, J=3.5 \mathrm{~Hz}, 2 \mathrm{H}, 2 \times 12-\mathrm{H}), 3.31$ $(d q, J=13.4,6.8 \mathrm{~Hz}, 2 \mathrm{H}, 2 \times 31-\mathrm{H}), 3.25-3.18(d d, J$ $=11.2,4.6 \mathrm{~Hz}, 2 \mathrm{H}, 2 \times 3-\mathrm{H}), 3.01(d q, J=12.8,6.5$ $\mathrm{Hz}, 2 \mathrm{H}, 2$ x 31-H), 2.02-1.98 (m, 2H 2 x 16-H), 1.98$1.94(m, 2 \mathrm{H}, 2 \times 11-\mathrm{H}), 1.93(d, J=3.3 \mathrm{~Hz}, 2 \mathrm{H}, 2 \times$ $18-\mathrm{H}), 1.87(d t, J=11.0,2.9 \mathrm{~Hz}, 2 \mathrm{H}, 2 \times 22-\mathrm{H}), 1.78$ $1.50(m, 20 \mathrm{H}, 2 \times 1-\mathrm{H}+2 \times 2-\mathrm{H}+2 \times 9-\mathrm{H}+2 \times$ $11-\mathrm{H}+2 \times 16-\mathrm{H}+2 \times 34-\mathrm{H}+2 \times 35-\mathrm{H}), 1.44(\mathrm{~m}, 12 \mathrm{H}$, $2 \times 15-\mathrm{H}+2 \times 7-\mathrm{H}+2 \times 6-\mathrm{H}+2 \times 21-\mathrm{H}+2 \times 22-\mathrm{H}$ $+2 \times 19-\mathrm{H}), 1.40-1.25(m, 6 \mathrm{H}, 2 \times 6-\mathrm{H}+2 \times 7-\mathrm{H}+$ $2 \times 21-\mathrm{H}), 1.10(s, 6 \mathrm{H}, 2 \times 27-\mathrm{H}), 1.08-1.00(m, 4 \mathrm{H}$, $2 \times 1-\mathrm{H}+2 \times 15-\mathrm{H}), 0.99(s, 6 \mathrm{H}, 2 \times 23-\mathrm{H}), 0.95$ $(s, 6 \mathrm{H}, 2 \times 30-\mathrm{H}), 0.92(s, 6 \mathrm{H}, 2 \times 25-\mathrm{H}), 0.87(s, 6 \mathrm{H}$,
$2 \times 26-\mathrm{H}), 0.78(s, 6 \mathrm{H}, 2 \times 24-\mathrm{H}), 0.78(s, 6 \mathrm{H}, 2 \times$ 29-H), 0.71 ( $m, 2 \mathrm{H}, 2$ x 5-H) ppm;

${ }^{13} \mathrm{C}$ NMR (125 MHz, $\left.\mathrm{CDCl}_{3}\right): \delta=178.1(2 \times \mathrm{C}-28)$, 140.0 ( $2 \times \mathrm{C}-13), 125.5$ ( $2 \times \mathrm{C}-12), 78.9(2 \times \mathrm{C}-3)$, 55.1 ( 2 x C-5), 53.9 ( 2 x C-18), 47.7 ( 2 x C-17), 47.5 (2 x C-9), 42.5 (2 x C-14), 39.7 (2 x C-8), 39.5 ( 2 x C-19), 39.2 (2 x C-31), 39.1 (2 x C-20), 38.7 ( 2 x C-1), 38.6 ( 2 x C-4), 37.2 ( 2 x C-22), 36.9 ( 2 x C-10), 32.7 ( 2 x C-7), 30.8 ( 2 x C-21), 28.1 ( 2 x C-23), 27.8 (2 x C-15), 27.1 ( 2 x C-32), 26.4 ( 2 x C-33), 24.8 ( 2 x C-2), 24.5 ( $2 \times$ C-16), 23. ( $2 \times \mathrm{C}-27), 23.2(2 \times \mathrm{C}-2)$, 21.2 ( 2 x C-30), 18.2 ( 2 x C-6), 17.2 ( 2 x C-24), 16.9 (2 x C-29), 15.6 (2 x C-26), 15.5 ( 2 x C-25) ppm; MS (ESI, $\mathrm{MeOH}): m / z=994.7\left(100 \%,[\mathrm{M}+\mathrm{H}]^{+}\right)$, $1017.6\left(90 \%,[\mathrm{M}+\mathrm{Na}]^{+}\right)$;

analysis calcd for $\mathrm{C}_{66} \mathrm{H}_{108} \mathrm{~N}_{2} \mathrm{O}_{4}$ (993.58): C 79.78, H 10.96, N 2.82; found: C 79.51, H 11.15, N 2.69.

\section{$\mathbf{N}^{1}, \mathbf{N}^{7}$-Bis-[(3ß)-3-hydroxy-urs-12-en-28-oyl]-1,7- diaminoheptane (26)}

Compound $26\left(0.05\right.$ g, 68\%) showed: m.p. $188^{\circ} \mathrm{C}$; $\mathrm{R}_{F}=0.3$ (silica gel, toluene/ethyl acetate/formic $\mathrm{acid} /$ heptane, 80:26:5:1); $[\alpha]_{\mathrm{D}}=+30.29^{\circ}$ (c 0.30, $\mathrm{CHCl}_{3}$ );

IR (KBr): $v=3406 b r, 2963 m, 2925 w, 1641 m, 1519 w$, $1455 w, 1377 w, 1260 w, 1260 s, 1088 s, 1016 s, 865 w$ $\mathrm{cm}^{-1}$;

${ }^{1} \mathrm{H}$ NMR $\left(500 \mathrm{MHz}, \mathrm{CDCl}_{3}\right): \delta=5.90(t, J=5.4 \mathrm{~Hz}$, $2 \mathrm{H}, 2 \times \mathrm{NH}), 5.30(t, J=3.6 \mathrm{~Hz}, 2 \mathrm{H}, 2 \times 12-\mathrm{H}), 3.31$ $(d q, J=13.6,7.0 \mathrm{~Hz}, 2 \mathrm{H}, 2 \times 31-\mathrm{H}), 3.22(d d, J=$ 11.2, $4.7 \mathrm{~Hz}, 2 \mathrm{H}, 2 \times 3-\mathrm{H}), 2.99(d q, J=12.7,7.0 \mathrm{~Hz}$, $2 \mathrm{H}, 2 \times 31-\mathrm{H}), 1.99(d, J=3.5 \mathrm{~Hz}, 2 \mathrm{H} 2 \times 16-\mathrm{H}), 1.96$ $(m, 2 \mathrm{H}, 2 \times 11-\mathrm{H}), 1.92(t d, J=16.7,3.4 \mathrm{~Hz}, 2 \mathrm{H}, 2 \times$ $18-\mathrm{H}), 1.89-1.83(m, 2 \mathrm{H}, 2 \times 22-\mathrm{H}), 1.78-1.50$ $(m, 20 \mathrm{H}, 2 \times 1-\mathrm{H}+2 \times 2-\mathrm{H}+2 \times 9-\mathrm{H}+2 \times 11-\mathrm{H}+2 \times$ $16-\mathrm{H}+2 \times 34-\mathrm{H}+2 \times 35-\mathrm{H}+36-\mathrm{H}), 1.49-1.41$ $(m, 12 \mathrm{H}, 2 \times 15-\mathrm{H}+2 \times 7-\mathrm{H}+2 \times 6-\mathrm{H}+2 \times 21-\mathrm{H}+$ $2 \times 22-\mathrm{H}+2 \times 19-\mathrm{H}), 1.40-1.25(m, 6 \mathrm{H}, 2 \times 6-\mathrm{H}+2$ x 7-H + 2 x 21-H), $1.10(s, 6 \mathrm{H}, 2 \times 27-\mathrm{H}), 1.08-1.00$ $(m, 4 \mathrm{H}, 2 \times 1-\mathrm{H}+2 \times 15-\mathrm{H}), 0.99(s, 6 \mathrm{H}, 2 \times 23-\mathrm{H})$, $0.95(s, 6 \mathrm{H}, 2 \times 30-\mathrm{H}), 0.93(s, 6 \mathrm{H}, 2 \times 25-\mathrm{H}), 0.87$ $(s, 6 \mathrm{H}, 2 \times 26-\mathrm{H}), 0.78(s, 6 \mathrm{H}, 2 \times 24-\mathrm{H}), 0.78(s, 6 \mathrm{H}$, $2 \times 29-\mathrm{H}), 0.71(\mathrm{~m}, 2 \mathrm{H}, 2 \times 5-\mathrm{H}) \mathrm{ppm}$;

${ }^{13} \mathrm{C}$ NMR (125 MHz, $\left.\mathrm{CDCl}_{3}\right): \delta=178.1(2 \times \mathrm{C}-28)$, $140.0(2 \times \mathrm{C}-13), 125.5$ ( $2 \times \mathrm{C}-12), 78.9(2 \times \mathrm{C}-3)$, 55.1 ( 2 x C-5), 54.0 (2 x C-18), 47.6 ( 2 x C-17), 47.5 (2 x C-9), 42.5 (2 x C-14), 39.7 (2 x C-8), 39.5 (2 x C-19), 39.4 ( 2 x C-31), 39.1 ( 2 x C-20), 38.7 ( 2 x C-1), 38.6 ( 2 x C-4), 37.1 ( 2 x C-22), 36.9 ( 2 x C-10), 32.7 ( 2 x C-7), 30.9 ( 2 x C-21), 29.2 (C-34), 28.9 ( 2 x C-33), 28.1 ( 2 x C-23), 27.8 ( 2 x C-15), 27.1 ( 2 x C-32), 24.8 ( $2 \times \mathrm{C}-2), 24.4$ ( $2 \times \mathrm{C}-16), 23.4$ ( $2 \times$ C-27), 23.2 ( $2 \times$ C-2), 21.2 ( $2 \times$ C-30), 18.2 ( 2 x C-6), 17.2 ( 2 x C-24), 16.9 ( 2 x C-29), 15.6 ( 2 x C-26), 15.5 (2 x C-25) ppm;

MS (ESI, MeOH): $m / z=1008.9\left(55 \%,[\mathrm{M}+\mathrm{H}]^{+}\right)$, $1030.9\left(30 \%,[\mathrm{M}+\mathrm{Na}]^{+}\right)$;

analysis calcd for $\mathrm{C}_{67} \mathrm{H}_{110} \mathrm{~N}_{2} \mathrm{O}_{4}$ (1007.60): C 79.86, H 11.00, N 2.78; found: C 79.66, H 11.18, N 2.67. 


\section{Acknowledgments}

Many thanks are due to Dr. D Ströhl and his team for the NMR spectra and to Dr. R. Kluge for the MSmeasurements. IR- and UV-vis spectra and optical rotations were recorded by Ms V. Simon. Financial support by the "ScienceCampus Halle, WCH" (W13004216 to R.C.) is gratefully acknowledged. The authors declare no conflict of interests.

\section{References}

1- M. Vrettou, A.A. Gray, A.R.E. Brewer, A.G.M. Barrett, Strategies for the synthesis of C-2 symmetric natural products- a review, Tetrahedron 2007, 63, 1487-1536.

2- G. Berube, Natural and synthetic biologically active dimeric molecules: Anticancer agents, anti-HIV agents, steroid derivatives and opioid antagonists, Curr. Med. Chem. 2006, 13, 131-154.

3- G. Berube, D. Rabouina, V. Perron, B. N'Zemba, R.C. Gaudreault, S. Parenta, E.E. Asselin, Synthesis of unique 17 beta-estradiol homo-dimers, estrogen receptors binding affinity evaluation and cytocidal activity on breast, intestinal and skin cancer cell lines, Steroids 2006, 71, 911-921.

4- T. Wezeman, K.S. Masters, S. Bräse, Double Trouble-The Art of Synthesis of Chiral Dimeric Natural Products, Angew. Chem. Int. Ed. 2014, 53, 4524-4526.

5- J. L. Jeffrey, R. Sarpong, An approach to the synthesis of dimeric resveratrol natural products via a palladium-catalysed domino reaction, Tetrahedron Lett. 2009, 50, 19691972.

6- N. J. Marianayagam, M. Sunde, J.M. Matthews, The power of two: protein dimerization in biology, Trends Biochem. Sci. 2004, 29 , 618-625.

7- B. Bednarczyk-Cwynar, A. Gunther, Advances in Chemistry and Pharmacology of Triterpenoid Synthetic Dimers, Curr. Med. Chem. 2017, 24, 2205-2240.

8- O. B. Kazakova, G.V. Giniyatullina, G.A. Tolstikov, V.E. Kataev, R.Z. Musin, Synthesis and modification of triterpenoids with two lupan backbones, Russ. J. Bioorg. Chem. 2009, 35, 645-650.

9- G. R. Pettit, N. Melody, F. Hempenstall, J.C. Chapuis, T.L. Groy, L. Williams, Antineoplastic Agents. 595. Structural Modifications of Betulin and the X-ray Crystal Structure of an Unusual Betulin Amine Dimer, J. Nat. Prod. 2014, 77, 863-872.

10- J. Hu, R.F. Li, J.R. Lu, Y. Ju, Synthesis and anion recognition of a novel oleanolic acidbased cyclic dimer, Tetrahedron Lett. 2011, 52, 4211-4214.

11- L. J. Dolby, M. Debono, Studies of Terpene Chemistry .1. Acid-Catalyzed Dimerization of Citronellal, J. Org. Chem. 1964, 29, 2306-2310.
12- C. M. Ma, N. Nakamura, M. Hattori, Chemical modification of oleanene type triterpenes and their inhibitory activity against HIV-1 protease dimerization, Chem. Pharm. Bull. 2000, 48, 1681-1688.

13- K. G. Cheng, J. Liu, H.B. Sun, J. Xie, Synthesis of Oleanolic Acid Dimers as Inhibitors of Glycogen Phosphorylase, Chem. Biodivers. 2010, 7, 690-697.

14- K. G. Cheng, C.H. Su, L.D. Yang, J. Liu, Z.F. Chen, Synthesis of oleanolic acid dimers linked at C-28 and evaluation of anti-tumor activity, Eur. J. Med. Chem. 2015, 89, 480-489.

15- J. R. Lu, J.D. Wu, Y. Ju, Tuning the aggregation mode to induce different chiralities in organogels of mono- and bis-triterpenoid derivatives and the preparation of gold nanoparticles for use as a template, New. J. Chem. 2014, 38, 6050-6056.

16- T. Honda, E.M. Padegimas, E. David, C. Sundararajan, K.T. Liby, C. Williams, M.B. Sporn, M. Visnick, 2-Cyano-3,10-dioxooleana1,9(11)-dien-28-oic acid anhydride. A novel and highly potent anti-inflammatory and cytoprotective agent, Bioorg. Med. Chem. Lett. 2010, 20, 2275-2278.

17- M. R. Prausnitz, R. Langer, Transdermal drug delivery, Nat. Biotechnol. 2008, 26, 1261-1268.

18- M. Kahnt, L. Fischer, A. Al-Harrasi, R. Csuk, Ethylenediamine Derived Carboxamides of Betulinic and Ursolic Acid as Potential Cytotoxic Agents, Molecules 2018, 23, 2558; doi:10.3390/molecules23102558.

19- M. Kahnt, L. Heller, P. Grabandt, A. AlHarrasi, R. Csuk, Platanic acid: A new scaffold for the synthesis of cytotoxic agents, Eur. J. Med. Chem. 2018, 143, 259-265.

20- M. Kahnt, J. Wiemann, L. Fischer, S. Sommerwerk, R. Csuk, Transformation of asiatic acid into a mitocanic, bimodal-acting rhodamine B conjugate of nanomolar cytotoxicity, Eur. J. Med. Chem. 2018, 159, 143-148.

21- L. Heller, A. Knorrscheidt, F. Flemming, J. Wiemann, S. Sommerwerk, I.Z. Pavel, A. AlHarrasi, R. Csuk, Synthesis and proapoptotic activity of oleanolic acid derived amides, Bioorg. Chem. 2016, 68, 137-151.

22- S. Sommerwerk, L. Heller, C. Kerzig, A.E. Kramell, R. Csuk, Rhodamine B conjugates of triterpenoic acids are cytotoxic mitocans even at nanomolar concentrations, Eur. J. Med. Chem. 2017, 127, 1-9.

23- S. Sommerwerk, L. Heller, J. Kuhfs, R. Csuk, Selective killing of cancer cells with triterpenoic acid amides - The substantial role of an aromatic moiety alignment, Eur. J. Med. Chem. 2016, 122, 452-464.

24- A. Loesche, M. Kahnt, I. Serbian, W. Brandt, R. Csuk, Triterpene-based carboxamides act as good inhibitors of butyrylcholinesterase, Molecules 2019, 24, 948/941-948/916. 
25- R. K. Wolfram, L. Fischer, R. Kluge, D. Stroehl, A. Al-Harrasi, R. Csuk, Homopiperazine-rhodamine B adducts of triterpenoic acids are strong mitocans, Eur. J. Med. Chem. 2018, 155, 869-879.

26- R. K. Wolfram, L. Heller, R. Csuk, Targeting mitochondria: Esters of rhodamine B with triterpenoids are mitocanic triggers of apoptosis, Eur. J. Med. Chem. 2018, 152, 21-30.

27- W. Jiang, R.-Z. Huang, J. Zhang, T. Guo, M.-T. Zhang, X.-C. Huang, B. Zhang, Z.-X. Liao, J. Sun, H.-S. Wang, Discovery of antitumor ursolic acid long-chain diamine derivatives as potent inhibitors of NF- $\mathrm{B}$, Bioorg. Chem. 2018, 79, 265-276.

28- L.-H. Zhang, Z.-H. Zhang, M.-Y. Li, Z.-Y. Wei, X.-J. Jin, H.-R. Piao, Synthesis and evaluation of the HIF- $1 \alpha$ inhibitory activities of novel ursolic acid tetrazole derivatives, Bioorg. Med. Chem. Lett. 2019, 29, 1440-1445.
29- L. Ruzicka, K. Hofmann, Polyterpenes and polyterpenoids. C. Transpositions in the rings A and $\mathrm{E}$ of oleanolic acid. Carbon skeleton of pentacyclic triterpenes, Helv. Chim. Acta 1936, 19, 114-128.

30- S. C. B. Gnoatto, L. Dalla Vechia, C.L. Lencina, A. Dassonville-Klimpt, S. Da Nascimento, D. Mossalayi, J. Guillon, G. Gosmann, P. Sonnet, Synthesis and preliminary evaluation of new ursolic and oleanolic acids derivatives as antileishmanial agents, J. Enzyme Inhib. Med. Chem. 2008, 23, 604-610.

31- M. Miyazawa, Y. Okuno, K. Imanishi, Suppression of the SOS-Inducing Activity of Mutagenic Heterocyclic Amine, Trp-P-1, by Triterpenoid from Uncaria sinensis in the Salmonella typhimurium TA1535/pSK1002 Umu Test, J. Agric. Food Chem. 2005, 53, 2312-2315. 